\title{
A Class of Variational-Hemivariational Inequalities in Reflexive Banach Spaces
}

\author{
Stanisław Migórski ${ }^{1}$ Anna Ochal $^{1}$ (D) Mircea Sofonea $^{2}$
}

Received: 26 February 2016 / Published online: 10 October 2016

(C) The Author(s) 2016. This article is published with open access at Springerlink.com

\begin{abstract}
We study a new class of elliptic variational-hemivariational inequalities in reflexive Banach spaces. An inequality in the class is governed by a nonlinear operator, a convex set of constraints and two nondifferentiable functionals, among which at least one is convex. We deliver a result on existence and uniqueness of a solution to the inequality. Next, we show the continuous dependence of the solution on the data of the problem and we introduce a penalty method, for which we state and prove a convergence result. Finally, we consider a mathematical model which describes the equilibrium of an elastic body in unilateral contact with a foundation. The model leads to a variational-hemivariational inequality for the displacement field, that we analyse by using our abstract results.
\end{abstract}

Keywords Variational-hemivariational inequality $\cdot$ Clarke subdifferential $\cdot$ Existence and uniqueness · Continuous dependence $\cdot$ Penalty operator $\cdot$ Frictional contact

Mathematics Subject Classification 47J20 - 47J22 - 49J53 - 74M10 · 74M15

\section{Introduction}

The theory of variational inequalities is traced back to early sixties when it was created and initially developed to deal with the Signorini problem. This theory can be considered as the fruitful interaction of elasticity theory and mathematical analysis, and since then it has gone through substantial development, see for instance [1-3, 17, 25] and the references therein. It

Research supported by the National Science Center of Poland under the Maestro Advanced Project No. DEC-2012/06/A/ST1/00262

A. Ochal

ochal@ii.uj.edu.pl

1 Faculty of Mathematics and Computer Science, Jagiellonian University in Krakow, ul. Łojasiewicza 6, 30348 Krakow, Poland

2 Laboratoire de Mathématiques et Physique, Université de Perpignan Via Domitia, 52 Avenue Paul Alduy, 66860 Perpignan, France 
was built on arguments of monotonicity and convexity, including properties of the subdifferential of a convex function. On the other hand, the theory of hemivariational inequalities has started in early eighties with the pioneering works of Panagiotopoulos [24-26] and is based on properties of the Clarke subdifferential, defined for locally Lipschitz functions which may be nonconvex. Analysis of hemivariational inequalities, including existence and uniqueness results, can be found in $[13,21,23,26]$. Both variational and hemivariational inequalities have been intensively used in the study of various problems in Mechanics, Physics and Engineering Sciences and, in particular, in Contact Mechanics. References on this matter include $[10,12,14,16,20,21,25,26,28,29,31]$, among others. Variational-hemivariational inequalities represent a special class of inequalities, in which both convex and nonconvex functions are involved. The interest in their study being motivated by various problems in Mechanics, as explained in [23] and the references therein.

We note that several classes of variational-hemivariational inequalities have been studied very recently in the following contributions. Some of them extend results which can be found in the monograph [23]. In [5] the author obtained results on existence of barrier solutions for multivalued variational inequalities including variational-hemivariational inequalities as special case. We refer to [6] to a survey paper which provides an analytical framework that allows to present in a unifying way and to extend a number of recent results. Paper [19] studies an interesting class of generalized quasi-variational hemivariational inequalities which involve multivalued mapping, and delivers results on existence of solution to optimal control problems. The existence of solutions for the variational-hemivariational inequalities in reflexive Banach spaces has been delivered in [32]. There, using the notion of the stable-quasimonotonicity some existence results are proved when the constrained set is nonempty, bounded or unbounded, closed and convex. Furthermore, we refer to [15] for existence results for elliptic and evolutionary variational and quasi-variational inequalities involving different types of pseudomonotone operators and a recessivity assumption that extends the classical coercivity conditions.

In [11] we have studied variational-hemivariational inequalities of the form: find $u \in V$ such that

$$
\begin{aligned}
& \langle A u, v-u\rangle+\int_{\Gamma}(F u)(\theta(\gamma v)-\theta(\gamma u)) d \Gamma \\
& \quad+j^{0}(u ; v-u) \geq\langle f, v-u\rangle \quad \text { for all } v \in V .
\end{aligned}
$$

These inequalities have been formulated in a particular functional framework, since in (1) we assumed that $\Gamma \subseteq \partial \Omega$ is a measurable part of the boundary of an open bounded subset $\Omega$ of $\mathbb{R}^{d}$ (with $d$ being a positive integer), $V$ is a closed subspace of $H^{1}\left(\Omega ; \mathbb{R}^{s}\right.$ ) (with $s \geq 1$ ), $F: V \rightarrow L^{2}(\Gamma)$ and $\theta: \mathbb{R}^{s} \rightarrow \mathbb{R}$ are Lipschitz continuous with constants $L_{F}$ and $L_{\theta}$, respectively, $F v \geq 0$ for all $v \in V, \theta$ is convex, and $\gamma: V \rightarrow L^{2}\left(\Gamma ; \mathbb{R}^{s}\right)$ denotes the trace operator. For these inequalities we proved an existence and uniqueness result of the solution, based on arguments of surjectivity for pseudomonotone operators and the Banach fixed point argument. Then, we studied the continuous dependence of the solution with respect to the data and we proved a convergence result. We also introduced a finite element discrete scheme for the numerical approximation of the inequalities, we showed their unique solvability, and we derived error estimates. In addition, we considered an example of frictional contact problem with normal compliance which leads to a variational-hemivariational inequality of the form (1) where the unknown is the displacement field. We applied our abstract results in the study of this problem and we obtained existence, uniqueness, convergence and error estimate results. Finally, we studied a history-dependent version of variational-hemivariational inequality (1), in our recent paper [30]. 
In this paper we continue the study of elliptic variational-hemivariational inequalities, extending part of our results obtained in [11] to variational-hemivariational inequalities with unilateral constraints, in the abstract framework of reflexive Banach spaces. For such inequalities there is a need to use arguments different to those used in [11]. The analysis of this new class of variational-hemivariational inequalities, including their unique solvability and the continuous dependence of the solution on the data, represents one of the traits of novelty of this paper. It also contains a convergence result that we state and prove by using a penalty method. Considering such kind of inequalities lays the functional background in the study of elastic contact problems with unilateral constraints and nonmonotone interface laws. To illustrate this statement, we introduce a new model of contact different to that considered in [11], for which we apply our abstract results.

The paper is organized as follows. In Sect. 2 we give a simple example which provides the physical motivation of our abstract study. Then, in Sect. 3 we fix the notation and recall preliminary material from nonlinear analysis. The class of variational-hemivariational inequalities is introduced in Sect. 4 where we state and prove an abstract existence and uniqueness result. Its proof is based, again, on arguments of surjectivity for pseudomonotone operators and the Banach fixed point theorem, but with a choice of spaces and operators different to that used in [11]. Next, in Sect. 5 we investigate the continuous dependence of the solution with respect to the data. In Sect. 6 we introduce a class of penalized problems, prove their unique solvability and the convergence of the corresponding solutions to the solution of the original problem, as the penalty parameter converges to zero. Finally, in Sect. 7 we deal with a unilateral contact problem for elastic materials in which the frictional contact conditions are in a subdifferential form. This contact problem is expressed as a variational-hemivariational inequality for the displacement field, for which we apply our abstract results, including the convergence theorem associated to the penalty method.

\section{A Spring-Rod System with Unilateral Constraints}

The abstract results we present in this paper are useful in the study of various mathematical models which describe the equilibrium of elastic bodies in frictional contact with a foundation. In Sect. 7 we shall present an example which illustrate the applicability of those results. Nevertheless, to provide the reader the physical motivation of our abstract study, we start with a very simple one-dimensional example which we present in what follows.

Consider an elastic rod which occupies the interval $[0, L]$ on the $O x$ axis. The rod is fixed at its end $x=0$ and its extremity $x=L$ is situated at the distance $g$ from a rigid obstacle. The gap between the rod and the obstacle is filled in two nonlinear springs $S_{1}$ and $S_{2}$ which are attached both to the rod and the obstacle. The natural length of the springs is $g$, and the system is in equilibrium when no forces are acting on the rod. This physical setting in depicted in Fig. 1. Assume now that the rod is submitted to the action of a body force of density $f_{0}$, which acts along the $O x$. Then, the problem of finding the equilibrium of the rod in the physical setting above can be formulated as follows.

Problem 1 Find a displacement field $u:[0, L] \rightarrow \mathbb{R}$ and a stress field $\sigma:[0, L] \rightarrow \mathbb{R}$ such that

$$
\sigma(x)=E \frac{d u}{d x}(x) \quad \text { for } x \in(0, L)
$$


$\mathrm{O}$

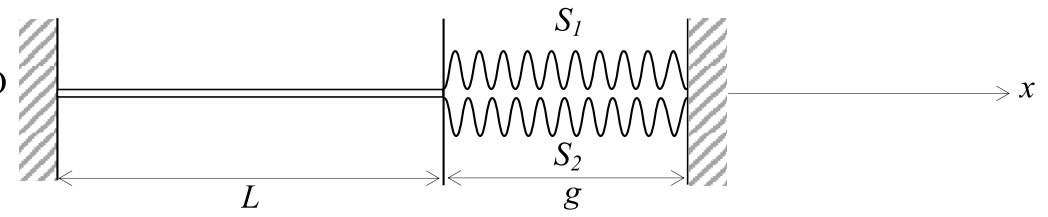

Fig. 1 The rod-spring system with unilateral constraints

$$
\begin{aligned}
& \frac{d \sigma}{d x}(x)+f_{0}(x)=0 \quad \text { for } x \in(0, L), \\
& u(0)=0, \\
& \begin{cases}u(L) \leq g, \\
-\sigma(L)=p_{1}(u(L))+p_{2}(u(L)) & \text { if } u(L)<g, \\
-\sigma(L) \geq p_{1}(u(L))+p_{2}(u(L)) & \text { if } u(L)=g .\end{cases}
\end{aligned}
$$

A brief description of the equations and conditions in Problem 1 is the following. First, (2) represents the elastic constitutive law in which $E>0$ is the Young modulus of the material and the derivative $\frac{d u}{d x}$ represents the linearized strain field. Equation (3) is the equilibrium equation and condition (4) represents the displacement condition. We use it here since the rod is assumed to be fixed at the end $x=0$. Conditions (5) represent the contact conditions in which our interest is and which we describe in detail.

Inequality $u(L) \leq g$ shows that the displacement in $x=L$ is subjected to unilateral constraint. This constraint arises from the physical setting, since the obstacle is assumed to be rigid and, therefore, its penetration is not allowed. Equality $u(L)=g$ corresponds to the case when the springs are completely compressed. Next, condition

$$
-\sigma(L)=p_{1}(u(L))+p_{2}(u(L)) \quad \text { if } u(L)<g
$$

shows that when the springs are partially compressed, then the stress in $x=L$ depends only on the displacement field in $x=L$ and has an additive decomposition. Here, $p_{i}$ are given continuous functions which describe the reaction of the springs $S_{i}$, for $i=1,2$, and are positive for a positive argument and negative for a negative argument. Such kind of decomposition is natural since the springs are connected in parallel. In addition, when the springs are in compression (i.e., when $0<u(L)<g$ ) then their reaction is in the negative direction of the $O x$ axis (since $\sigma(L)<0$ ) and when the springs are in extension (i.e., when $u(L)<0$ ), then their reaction is in the positive orientation of the $O x$ axis (since $\sigma(L)>0$ ).

Assume now that $u(L)=g$, i.e., the springs are totally compressed. Then (5) implies that

$$
\sigma(L)+p_{1}(u(L))+p_{2}(u(L)) \leq 0
$$

and, moreover, the properties of the functions $p_{1}$ and $p_{2}$ show that

$$
-p_{1}(u(L))-p_{2}(u(L)) \leq 0 .
$$

Therefore,

$$
\sigma(L)=\sigma_{e}(L)+\sigma_{a}(L),
$$


where

$$
\begin{aligned}
& \sigma_{e}(L)=\sigma(L)+p_{1}(u(L))+p_{2}(u(L)) \leq 0, \\
& \sigma_{a}(L)=-p_{1}(u(L))-p_{2}(u(L)) \leq 0 .
\end{aligned}
$$

We conclude from (6)-(8) that, when the springs are totally compressed, then the pressure exerted in $x=L, \sigma(L)$, is decomposed into two parts: an elastic one, $\sigma_{e}(L)$, and an additional one, $\sigma_{a}(L)$, both negative. The additional pressure prevents the displacement of the extremity $x=L$ of the rod in such a way that the constraint $u(L) \leq g$ is satisfied.

In the study of Problem 1 we denote by $q_{i}: \mathbb{R} \rightarrow \mathbb{R}$ the functions defined by

$$
q_{i}(r)=\int_{0}^{r} p_{i}(s) d s \quad \text { for all } r \in \mathbb{R},
$$

for $i=1,2$. We assume that $p_{1}$ is an increasing function and, therefore, $q_{1}$ is a convex function. Moreover, the following inequality holds

$$
q_{1}(r)-q_{1}(s) \geq p_{1}(s)(r-s) \quad \text { for all } r, s \in \mathbb{R} .
$$

In contrast, the function $p_{2}$ is not assumed to be monotone and this represents the main novelty of our rod-spring model. As a consequence, the function $q_{2}$ could be nonconvex. Nevertheless, it satisfies the equality

$$
q_{2}^{0}(s ; r)=p_{2}(s) r \quad \text { for all } r, s \in \mathbb{R},
$$

where $q_{2}^{0}(s ; r)$ denotes the generalized directional derivative of $q_{2}$ at the point $s$ in the direction $r$, see Definition 8 below.

We use the space

$$
X=\left\{v \in H^{1}(0, L) \mid v(0)=0\right\}
$$

which is a real Hilbert space with the canonical inner product. We denote by $X^{*}$ and $\langle\cdot, \cdot\rangle$ the dual of $X$ and the duality pairing between $X^{*}$ and $X$, respectively. We assume that $f_{0} \in L^{2}(0, L)$ and we define the set $K$, the operator $A: X \rightarrow X^{*}$, the element $f \in X^{*}$ and the functions $\varphi: X \rightarrow \mathbb{R}, j: X \rightarrow \mathbb{R}$ by

$$
\begin{aligned}
& K=\{u \in V \mid u(L) \leq g\} \\
& \langle A u, v\rangle=\int_{0}^{L} E \frac{d u}{d x} \frac{d v}{d x} d x \quad \text { for all } u, v \in V, \\
& \varphi(v)=q_{1}(v(L)) \text { for all } v \in V \\
& j(v)=q_{2}(v(L)) \text { for all } v \in V, \\
& \langle f, v\rangle=\int_{0}^{L} f_{0} v d x \text { for all } v \in V .
\end{aligned}
$$

To derive the variational formulation of Problem 1 we assume in what follows that $(u, \sigma)$ are sufficiently smooth functions which satisfy (2)-(5) and let $v \in K$. First, we perform an integration by parts and use the equilibrium equation (3) to see that

$$
\int_{0}^{L} \sigma\left(\frac{d v}{d x}-\frac{d u}{d x}\right) d x=\int_{0}^{L} f_{0}(v-u) d x+\sigma(L)(v(L)-u(L))-\sigma(0)(v(0)-u(0)) .
$$


Next, since $v(0)=u(0)=0$, we deduce that

$$
\int_{0}^{L} \sigma\left(\frac{d v}{d x}-\frac{d u}{d x}\right) d x=\int_{0}^{L} f_{0}(v-u) d x+\sigma(L)(v(L)-u(L)) .
$$

Moreover, we write

$$
\begin{aligned}
\sigma(L)(v(L)-u(L))= & \left(\sigma(L)+p_{1}(u(L))+p_{2}(u(L))\right)(v(L)-g) \\
& +\left(\sigma(L)+p_{1}(u(L))+p_{2}(u(L))\right)(g-u(L)) \\
& -p_{1}(u(L))(v(L)-u(L))-p_{2}(u(L))(v(L)-u(L)),
\end{aligned}
$$

then we use the contact condition (5) and the definition (12) of the set $K$, to deduce that

$$
\sigma(L)(v(L)-u(L)) \geq-p_{1}(u(L))(v(L)-u(L))-p_{2}(u(L))(v(L)-u(L)) .
$$

We now combine (17), (18) and use (10), (11), (16) to find that

$$
\int_{0}^{L} \sigma\left(\frac{d v}{d x}-\frac{d u}{d x}\right) d x+q_{1}(v(L))-q_{1}(u(L))+q_{2}^{0}(u(L) ; v(L)-u(L)) \geq\langle f, v-u\rangle .
$$

On the other hand, a simple computation based on (15) and Definition 8 below shows that

$$
j^{0}(u ; v)=q_{2}^{0}(u(L) ; v(L)) \quad \text { for all } u, v \in V,
$$

where $j^{0}(u ; v)$ denotes the generalized directional derivative of $j$ at the point $u$ in the direction $v$. We now substitute the constitutive law (2) in (19), then we use definitions (13), (14) and equality (20) to obtain the following variational formulation of Problem 1: Find a displacement field $u \in K$ such that

$$
\langle A u, v-u\rangle+\varphi(v)-\varphi(u)+j^{0}(u ; v-u) \geq\langle f, v-u\rangle \text { for all } v \in K .
$$

Recall that $q_{1}$ is a convex function and, therefore, the function $\varphi$ is convex. On the other hand, the function $j$ could be nonconvex. We conclude from above that inequality (21) represents a variational-hemivariational inequality. This example motivates to consider inequalities of the form (21) in an abstract functional framework. Their analysis is performed in Sects. 4-6.

\section{Preliminaries}

In this part we recall the preliminary material on subdifferentials and monotone type operators which will be used in the next sections. For more details, we refer to several monographs, e.g., [7-9, 21, 23].

We use the following notation. Let $X$ be a real normed space with norm denoted by $\|\cdot\|_{X}$. By $\langle\cdot, \cdot\rangle$ we denote the duality pairing between $X^{*}$ and $X, X^{*}$ being topological dual to $X$. Let $0_{X}$ be zero element in $X$. The symbol $w-X$ is used for the space $X$ endowed with the weak topology while $2^{X^{*}}$ denotes the set of all subsets of $X^{*}$. Unless otherwise stated, we always assume that $X$ is a Banach space. 
For a multivalued operator $T: X \rightarrow 2^{X^{*}}$, its domain $D(T)$, range $R(T)$ and graph $G r(T)$ are defined, respectively, by

$$
\begin{aligned}
& D(T)=\{x \in X \mid T x \neq \emptyset\}, \quad R(T)=\bigcup\{T x \mid x \in X\}, \\
& G r(T)=\left\{\left(x, x^{*}\right) \in X \times X^{*} \mid x^{*} \in T x\right\} .
\end{aligned}
$$

We start with the following definition.

Definition 2 An operator $T: X \rightarrow 2^{X^{*}}$ is called monotone if $\left\langle u^{*}-v^{*}, u-v\right\rangle \geq 0$ for all $\left(u, u^{*}\right),\left(v, v^{*}\right) \in \operatorname{Gr}(T)$. It is maximal monotone, if it is monotone and maximal in the sense of inclusion of graphs in the family of monotone operators from $X$ to $2^{X^{*}}$. Operator $T$ is called coercive, if $\left\langle u^{*}, u\right\rangle \geq \alpha\left(\|u\|_{X}\right)\|u\|_{X}$ for all $\left(u, u^{*}\right) \in \operatorname{Gr}(T)$ with a function $\alpha: \mathbb{R}_{+} \rightarrow \mathbb{R}$ such that $\lim _{r \rightarrow+\infty} \alpha(r)=+\infty$. Furthermore, given $u_{0} \in X, T$ is said to be $u_{0}$-coercive, if there exists a function $\alpha: \mathbb{R}_{+} \rightarrow \mathbb{R}$ with $\lim _{r \rightarrow+\infty} \alpha(r)=+\infty$ such that for all $\left(u, u^{*}\right) \in G r(T)$, we have $\left\langle u^{*}, u-u_{0}\right\rangle \geq \alpha\left(\|u\|_{X}\right)\|u\|_{X}$.

Next, we recall the notions of pseudomonotonicity and generalized pseudomonotonicity for a multivalued operator.

Definition 3 Let $X$ be a reflexive Banach space. A multivalued operator $T: X \rightarrow 2^{X^{*}}$ is pseudomonotone if:

(a) for every $u \in X$, the set $T u \subset X^{*}$ is nonempty, closed and convex;

(b) $T$ is upper semicontinuous from each finite dimensional subspace of $X$ into $w$ - $X^{*}$;

(c) for any sequences $\left\{u_{n}\right\} \subset X$ and $\left\{u_{n}^{*}\right\} \subset X^{*}$ such that $u_{n} \rightarrow u$ weakly in $X, u_{n}^{*} \in T u_{n}$ for all $n \geq 1$ and $\limsup \left\langle u_{n}^{*}, u_{n}-u\right\rangle \leq 0$, we have that for every $v \in X$, there exists $u^{*}(v) \in T u$ such that

$$
\left\langle u^{*}(v), u-v\right\rangle \leq \liminf \left\langle u_{n}^{*}, u_{n}-v\right\rangle \text {. }
$$

Definition 4 Let $X$ be a reflexive Banach space. A multivalued operator $T: X \rightarrow 2^{X^{*}}$ is generalized pseudomonotone if for any sequences $\left\{u_{n}\right\} \subset X$ and $\left\{u_{n}^{*}\right\} \subset X^{*}$ such that $u_{n} \rightarrow u$ weakly in $X, u_{n}^{*} \in T u_{n}$ for $n \geq 1, u_{n}^{*} \rightarrow u^{*}$ weakly in $X^{*}$ and $\lim \sup \left\langle u_{n}^{*}, u_{n}-u\right\rangle \leq 0$, we have $u^{*} \in T u$ and

$$
\lim \left\langle u_{n}^{*}, u_{n}\right\rangle=\left\langle u^{*}, u\right\rangle .
$$

The relationship between these notions is given by the following result (cf. [9, Propositions 1.3.65 and 1.3.66]).

Proposition 5 Let $X$ be a reflexive Banach space and $T: X \rightarrow 2^{X^{*}}$.

(a) If $T$ is pseudomonotone, then it is generalized pseudomonotone.

(b) If $T$ is a bounded generalized pseudomonotone operator such that for all $u \in X, T u$ is a nonempty, closed and convex subset of $X^{*}$, then $T$ is pseudomonotone.

Given $u_{0} \in X$ and $T: X \rightarrow 2^{X^{*}}$, a multivalued operator $T_{u_{0}}$ is defined by $T_{u_{0}}(v)=T(v+$ $u_{0}$ ) for all $v \in X$. We recall the following surjectivity result which is a consequence of Theorem 2.12 in [23] and will be used in next sections. 
Theorem 6 Let $X$ be a reflexive Banach space, $T_{1}: X \rightarrow 2^{X^{*}}$ a pseudomonotone operator and $T_{2}: X \rightarrow 2^{X^{*}}$ a maximal monotone operator, and $u_{0} \in D\left(T_{2}\right)$. Assume that $T_{1}$ is $u_{0^{-}}$ coercive in the sense of Definition 2, and either $T_{1 u_{0}}$ or $T_{2 u_{0}}$ is bounded. Then $T_{1}+T_{2}$ is surjective, i.e., $R\left(T_{1}+T_{2}\right)=X^{*}$.

We hereafter recall the definitions of the convex and the Clarke subdifferentials.

Definition 7 Let $\varphi: X \rightarrow \mathbb{R} \cup\{+\infty\}$ be a proper, convex and lower semicontinuous function. The mapping $\partial \varphi: X \rightarrow 2^{X^{*}}$ defined by

$$
\partial \varphi(x)=\left\{x^{*} \in X^{*} \mid\left\langle x^{*}, v-x\right\rangle \leq \varphi(v)-\varphi(x) \text { for all } v \in X\right\}
$$

is called the subdifferential of $\varphi$. An element $x^{*} \in \partial \varphi(x)$ is called a subgradient of $\varphi$ in $x$.

Definition 8 Let $h: X \rightarrow \mathbb{R}$ be a locally Lipschitz function. The generalized (Clarke) directional derivative of $h$ at the point $x \in X$ in the direction $v \in X$ is defined by

$$
h^{0}(x ; v)=\limsup _{y \rightarrow x, \lambda \downarrow 0} \frac{h(y+\lambda v)-h(y)}{\lambda} .
$$

The generalized gradient (subdifferential) of $h$ at $x$ is a subset of the dual space $X^{*}$ given by

$$
\partial h(x)=\left\{\zeta \in X^{*} \mid h^{0}(x ; v) \geq\langle\zeta, v\rangle \text { for all } v \in X\right\} .
$$

A locally Lipschitz function $h$ is said to be regular (in the sense of Clarke) at the point $x \in X$ if for all $v \in X$ the one-sided directional derivative $h^{\prime}(x ; v)$ exists and $h^{0}(x ; v)=h^{\prime}(x ; v)$.

Finally, we recall several definitions for single-valued operators.

Definition 9 A single-valued operator $A: X \rightarrow X^{*}$ is said to be monotone, if for all $u$, $v \in X$, we have $\langle A u-A v, u-v\rangle \geq 0$. It is called maximal monotone, if it is monotone, and $\langle A u-w, u-v\rangle \geq 0$ for any $u \in X$ entails $w=A v$. Operator $A$ is called bounded, if $A$ maps bounded sets of $X$ into bounded sets of $X^{*}$. It is said to be coercive, if $\langle A u, u\rangle \geq$ $\alpha\left(\|u\|_{X}\right)\|u\|_{X}$ for all $u \in X$, where $\alpha: \mathbb{R}_{+} \rightarrow \mathbb{R}$ is a function such that with $\lim _{r \rightarrow+\infty} \alpha(r)=$ $+\infty$. Moreover, $A$ is called pseudomonotone, if it is bounded and $u_{n} \rightarrow u$ weakly in $X$ with $\limsup \left\langle A u_{n}, u_{n}-u\right\rangle \leq 0 \operatorname{imply}\langle A u, u-v\rangle \leq \liminf \left\langle A u_{n}, u_{n}-v\right\rangle$ for all $v \in X$.

Remark 10 It can be shown that an operator $A: X \rightarrow X^{*}$ is pseudomonotone, if and only if it is bounded and $u_{n} \rightarrow u$ weakly in $X$ together with $\lim \sup \left\langle A u_{n}, u_{n}-u\right\rangle \leq 0$ yield $\lim \left\langle A u_{n}, u_{n}-u\right\rangle=0$ and $A u_{n} \rightarrow A u$ weakly in $X^{*}$.

\section{An Existence and Uniqueness Result}

Let $X$ be a reflexive Banach space. Given an operator $A: X \rightarrow X^{*}$, functions $\varphi: K \times$ $K \rightarrow \mathbb{R}, j: X \rightarrow \mathbb{R}$ and a set $K \subset X$, we consider the following problem.

Problem 11 Find an element $u \in K$ such that

$$
\langle A u, v-u\rangle+\varphi(u, v)-\varphi(u, u)+j^{0}(u ; v-u) \geq\langle f, v-u\rangle \quad \text { for all } v \in K
$$


For the study of Problem 11, we need the following hypotheses on the data.

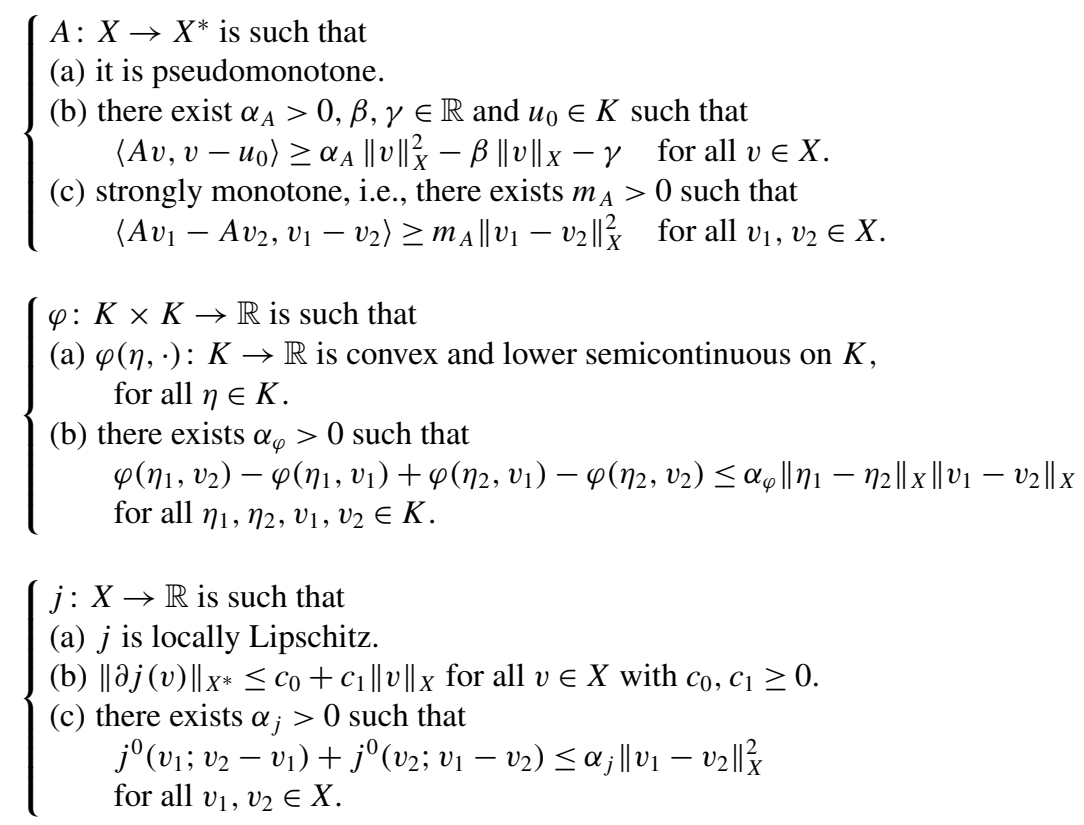

$K$ is nonempty, closed and convex subset of $X$.

$$
f \in X^{*} \text {. }
$$

In the statement of Problem 11 the function $\varphi(u, \cdot)$ is assumed to be convex and the function $j$ is locally Lipschitz and, in general, nonconvex. For this reason, inequality in Problem 11 is called a variational-hemivariational inequality. The motivation to study Problem 11 comes from the fact that it contains, as particular cases, various problems considered in the literature.

1. For $j \equiv 0$, Problem 11 reduces to the elliptic quasivariational inequality of the first kind of the form

$$
u \in K, \quad\langle A u, v-u\rangle+\varphi(u, v)-\varphi(u, u) \geq\langle f, v-u\rangle \quad \text { for all } v \in K
$$

studied in [31], for instance.

2. For $j \equiv 0$ and $K=X$, Problem 11 reduces to the elliptic quasivariational inequality of the second kind of the form

$$
u \in X, \quad\langle A u, v-u\rangle+\varphi(u, v)-\varphi(u, u) \geq\langle f, v-u\rangle \quad \text { for all } v \in X
$$

considered in [31].

3. For $j \equiv 0$ and $\varphi(u, v)=\varphi(v)$, Problem 11 takes the form of the elliptic variational inequality of the first kind of the form

$$
u \in K, \quad\langle A u, v-u\rangle+\varphi(v)-\varphi(u) \geq\langle f, v-u\rangle \quad \text { for all } v \in K
$$

treated in $[18,31]$. 
4. For $j \equiv 0, K=X$ and $\varphi(u, v)=\varphi(v)$, Problem 11 reduces to the elliptic variational inequality of the second kind of the form

$$
u \in X, \quad\langle A u, v-u\rangle+\varphi(v)-\varphi(u) \geq\langle f, v-u\rangle \quad \text { for all } v \in X
$$

studied in $[2,18,31]$.

5. For $j \equiv 0$ and $\varphi \equiv 0$, Problem 11 reduces to the elliptic variational inequality of the form

$$
u \in K, \quad\langle A u, v-u\rangle \geq\langle f, v-u\rangle \quad \text { for all } v \in K
$$

considered in $[2,4,17,18]$.

6. For $\varphi \equiv 0, K=X$, from Problem 11 , we obtain the elliptic hemivariational inequality of the form

$$
u \in X, \quad\langle A u, v\rangle+j^{0}(u ; v) \geq\langle f, v\rangle \quad \text { for all } v \in X
$$

investigated in [23].

7. For $j \equiv 0, \varphi \equiv 0$ and $K=X$, Problem 11 reduces to the elliptic equation

$$
u \in X, \quad A u=f .
$$

Remark 12 Note that the variational-hemivariational inequality (1) studied in [11] is of form in Problem 11 with $K=X=V$ and $\varphi(u, v)=\int_{\Gamma}(F u) \theta(\gamma v) d \Gamma$ for $u, v \in V$. In this case, hypothesis (23)(b) holds with $\alpha_{\varphi}=\|\gamma\| L_{F} L_{\theta}$.

Remark 13 We note that if $A: X \rightarrow X^{*}$ is strongly monotone, i.e., (22)(c) holds and

$$
\|A v\|_{X^{*}} \leq a_{0}+a_{1}\|v\|_{X} \quad \text { for all } v \in X
$$

with $a_{0}, a_{1} \geq 0$, then $A$ satisfies (22)(b) with constant $\alpha_{A}=\frac{1}{2} m_{A}$.

Remark 14 It can be proved that for a locally Lipschitz function $j: X \rightarrow \mathbb{R}$, hypothesis $(24)(c)$ is equivalent to the following condition:

$$
\left\langle\partial j\left(v_{1}\right)-\partial j\left(v_{2}\right), v_{1}-v_{2}\right\rangle \geq-\alpha_{j}\left\|v_{1}-v_{2}\right\|_{X}^{2} \quad \text { for all } v_{1}, v_{2} \in X .
$$

The latter is the so-called relaxed monotonicity condition and it was extensively used in the literature, cf., e.g., [21]. Note also that if $j: X \rightarrow \mathbb{R}$ is a convex function, then (24)(c) or, equivalently, condition (27) always holds since it reduces to the monotonicity of the (convex) subdifferential, i.e., $\alpha_{j}=0$.

We provide below some examples of functions which satisfy condition (24)(c). The multivalued subdifferential condition is obtained by applying the "filling in a gap procedure".

Example 15 Consider the function $j: \mathbb{R} \rightarrow \mathbb{R}$ defined by

$$
j(r)=\int_{0}^{r} p(s) d s \quad \text { for } r \in \mathbb{R},
$$

where $p \in L_{l o c}^{\infty}(\mathbb{R})$. For $r \in \mathbb{R}$, we define

$$
\underline{p}(r)=\lim _{\varepsilon \rightarrow 0^{+}} \operatorname{essinf} p(s) \quad \text { and } \quad \bar{p}(r)=\lim _{\varepsilon \rightarrow 0^{+}} \underset{|s-r|<\varepsilon}{\operatorname{ess} \sup } p(s) .
$$


It is clear that $j$ is locally Lipschitz and $\partial j(r) \subset[\underline{p}(r), \bar{p}(r)]$ for $r \in \mathbb{R}$. If additionally $p$ satisfies the condition

$$
\bar{p}\left(r_{1}\right) \leq \underline{p}\left(r_{2}\right)+M\left(r_{2}-r_{1}\right) \quad \text { for all } r_{1}, r_{2} \in \mathbb{R} \text { with } M \geq 0,
$$

then $j$ satisfies the relaxed monotonicity condition (24)(c) with $\alpha_{j}=M$.

The following two examples provide subdifferentials which are single-valued and multivalued, respectively.

Example 16 Let $p: \mathbb{R} \rightarrow \mathbb{R}$ be the function defined by

$$
p(r)= \begin{cases}0 & \text { if } r<0 \\ r & \text { if } 0 \leq r<1 \\ 2-r & \text { if } 1 \leq r<2 \\ \sqrt{r-2}+r-2 & \text { if } 2 \leq r<6 \\ r & \text { if } r \geq 6\end{cases}
$$

This function is continuous and nonconvex, and it is neither monotone, nor Lipschitz continuous. Define the function $j: \mathbb{R} \rightarrow \mathbb{R}$ by (28). Since $j^{\prime}(r)=p(r)$ for all $r \in \mathbb{R}$, it is clear that $j$ is a $C^{1}$ function, and thus it is locally Lipschitz. Since $|p(r)| \leq|r|$ for $r \in \mathbb{R}$, we know that $j$ satisfies (24)(b). Moreover, the function $\mathbb{R} \ni r \mapsto r+p(r) \in \mathbb{R}$ is nondecreasing which implies

$$
\left(p\left(r_{1}\right)-p\left(r_{2}\right)\right)\left(r_{2}-r_{1}\right) \leq\left(r_{1}-r_{2}\right)^{2} \text { for all } r_{1}, r_{2} \in \mathbb{R} .
$$

We combine this inequality with equality $j^{0}\left(r_{1} ; r_{2}\right)=p\left(r_{1}\right) r_{2}$ valid for all $r_{1}, r_{2} \in \mathbb{R}$, to see that condition (24)(c) is satisfied with $\alpha_{j}=1$. Hence, $j$ satisfies the hypothesis (24).

Example 17 Let $p: \mathbb{R} \rightarrow \mathbb{R}$ be the discontinuous function given by

$$
p(r)= \begin{cases}0 & \text { if } r<0, \\ e^{-r}+a & \text { if } r \geq 0\end{cases}
$$

where $a \geq 0$. Then the function $j$ defined by (28) has the form

$$
j(r)= \begin{cases}0 & \text { if } r<0 \\ -e^{-r}+a r+1 & \text { if } r \geq 0\end{cases}
$$

its subdifferential is given by

$$
\partial j(r)= \begin{cases}0 & \text { if } r<0 \\ {[0,1+a]} & \text { if } r=0 \\ e^{-r}+a & \text { if } r>0\end{cases}
$$

and $j$ satisfies condition (24)(c) with $\alpha_{j}=1$.

Further examples of nonconvex functions satisfying condition (24) can be found in [22].

Our existence and uniqueness result for Problem 11 is the following. 
Theorem 18 Assume (22)-(26) and, in addition, assume the smallness conditions

$$
\begin{aligned}
& \alpha_{\varphi}+\alpha_{j}<m_{A}, \\
& \alpha_{j}<\alpha_{A} .
\end{aligned}
$$

Then, Problem 11 has a unique solution $u \in X$.

Proof of Theorem 18 is carried out in several steps. In the rest of the section, we assume conditions (22)-(30). Let $\eta \in K$ be given and consider the following auxiliary problem.

Problem 19 Find $u_{\eta} \in X$ such that $u_{\eta} \in K$ and

$$
\left\langle A u_{\eta}, v-u_{\eta}\right\rangle+\varphi(\eta, v)-\varphi\left(\eta, u_{\eta}\right)+j^{0}\left(u_{\eta} ; v-u_{\eta}\right) \geq\left\langle f, v-u_{\eta}\right\rangle \quad \text { for all } v \in K
$$

We will prove the following result.

Lemma 20 Problem 19 has a unique solution $u_{\eta} \in X$.

Proof For the existence part, we apply Theorem 6 . We define $\widetilde{\varphi}_{\eta}: X \rightarrow \mathbb{R} \cup\{+\infty\}$ by

$$
\tilde{\varphi}_{\eta}(v)= \begin{cases}\varphi(\eta, v) & \text { if } v \in K \\ +\infty & \text { otherwise }\end{cases}
$$

for $v \in X$. Using this notation, Problem 19 is equivalent to: find $u_{\eta} \in X$ such that

$$
\left\langle A u_{\eta}, v-u_{\eta}\right\rangle+\widetilde{\varphi}_{\eta}(v)-\widetilde{\varphi}_{\eta}\left(u_{\eta}\right)+j^{0}\left(u_{\eta} ; v-u_{\eta}\right) \geq\left\langle f, v-u_{\eta}\right\rangle \quad \text { for all } v \in X
$$

Next, we consider the following problem: find $u_{\eta} \in X$ such that

$$
A u_{\eta}+\partial j\left(u_{\eta}\right)+\partial \widetilde{\varphi}_{\eta}\left(u_{\eta}\right) \ni f .
$$

We introduce two multivalued operators $T_{1}, T_{2}: X \rightarrow 2^{X^{*}}$ which appear in (33) and are defined by

$$
T_{1} v=A v+\partial j(v), \quad T_{2} v=\partial \widetilde{\varphi}_{\eta}(v) \quad \text { for } v \in X
$$

respectively.

We claim that the operator $T_{1}$ is bounded, $u_{0}$-coercive in the sense of Definition 2 and pseudomonotone.

The boundedness of the operator $T_{1}$ follows easily from the boundedness of $A$ and the growth condition (24)(b) on $\partial j$. In order to establish the $u_{0}$-coercivity of $T_{1}$, we use hypotheses (22)(b), (24)(c), Remark 14 and the following inequality which follows from (24)(b)

$$
\left|\left\langle\partial j\left(u_{0}\right), v-u_{0}\right)\right| \leq\left(c_{0}+c_{1}\left\|u_{0}\right\|_{X}\right)\left\|v-u_{0}\right\|_{X} .
$$

We have 


$$
\begin{aligned}
\left\langle T_{1} v, v-u_{0}\right\rangle= & \left\langle A v, v-u_{0}\right\rangle+\left\langle\partial j(v)-\partial j\left(u_{0}\right), v-u_{0}\right\rangle+\left\langle\partial j\left(u_{0}\right), v-u_{0}\right\rangle \\
\geq & \alpha_{A}\|v\|_{X}^{2}-\beta\|v\|_{X}-\gamma-\alpha_{j}\left\|v-u_{0}\right\|_{X}^{2}-\left(c_{0}+c_{1}\left\|u_{0}\right\|_{X}\right)\left\|v-u_{0}\right\|_{X} \\
\geq & \left(\alpha_{A}-\alpha_{j}\right)\|v\|_{X}^{2}-\|v\|_{X}\left(\beta+2 \alpha_{j}\left\|u_{0}\right\|_{X}+c_{0}+c_{1}\left\|u_{0}\right\|_{X}\right) \\
& \quad-\gamma-\alpha_{j}\left\|u_{0}\right\|_{X}^{2}-\left(c_{0}+c_{1}\left\|u_{0}\right\|_{X}\right)\left\|u_{0}\right\|_{X}
\end{aligned}
$$

for all $v \in X$. Taking into account hypothesis (30), the $u_{0}$-coercivity of $T_{1}$ follows.

We now prove that the operator $T_{1}$ is pseudomonotone. First, we observe that since for all $v \in X$, the set $A v+\partial j(v)$ is nonempty, closed and convex in $X^{*}$, it is enough to show, cf. Proposition 5, that $T_{1}$ is generalized pseudomonotone. Second, it is easy to see by hypotheses (22)(c), (24)(c) and (29), and Remark 14 that the operator $T_{1}$ is strongly monotone, i.e.,

$$
\left\langle T_{1} v_{1}-T_{1} v_{2}, v_{1}-v_{2}\right\rangle \geq\left(m_{A}-\alpha_{j}\right)\left\|v_{1}-v_{2}\right\|_{X}^{2} \quad \text { for all } v_{1}, v_{2} \in X .
$$

Next, in order to show that $T_{1}$ is generalized pseudomonotone, let $u_{n} \in X, u_{n} \rightarrow u$ weakly in $X, u_{n}^{*} \in T_{1} u_{n}, u_{n}^{*} \rightarrow u^{*}$ weakly in $X^{*}$ and $\lim \sup \left\langle u_{n}^{*}, u_{n}-u\right\rangle \leq 0$. We need to prove that $u^{*} \in T_{1} u$ and $\left\langle u_{n}^{*}, u_{n}\right\rangle \rightarrow\left\langle u^{*}, u\right\rangle$. Using the strong monotonicity of $T_{1}$, from the relation

$$
\left(m_{A}-\alpha_{j}\right)\left\|u_{n}-u\right\|_{X}^{2} \leq\left\langle u_{n}^{*}, u_{n}-u\right\rangle-\left\langle T_{1} u, u_{n}-u\right\rangle,
$$

we deduce that $u_{n} \rightarrow u$ in $X$. From $u_{n}^{*} \in T_{1} u_{n}$, we have $u_{n}^{*}=w_{n}+z_{n}$ with $w_{n}=A u_{n}$ and $z_{n} \in \partial j\left(u_{n}\right)$. Since $A$ and $\partial j$ are bounded operators, by passing to a subsequence, if necessary, we may assume that $w_{n} \rightarrow w$ and $z_{n} \rightarrow z$ both weakly in $X^{*}$ with some $w$, $z \in X^{*}$. Therefore, from $u_{n}^{*}=w_{n}+z_{n}$, we immediately have $u^{*}=w+z$. Exploiting the equivalent condition for the pseudomonotonicity of $A$ in Remark 10, we have $A u_{n} \rightarrow A u$ weakly in $X^{*}$, which gives $w=A u$. On the other hand, because $X \ni v \mapsto \partial j(v) \in 2^{X^{*}}$ has a closed graph with respect to the strong topology in $X$ and weak topology in $X^{*}$, we infer that $z \in \partial j(u)$. Hence, $u^{*}=w+z \in A u+\partial j(u)=T_{1} u$. Since $u_{n}^{*} \rightarrow u^{*}$ weakly in $X^{*}$ and $u_{n} \rightarrow u$ in $X$, it is clear that $\left\langle u_{n}^{*}, u_{n}\right\rangle \rightarrow\left\langle u^{*}, u\right\rangle$. This shows that $T_{1}$ is generalized pseudomonotone and also that $T_{1}$ is pseudomonotone, which completes the proof of the claim.

Finally, from hypothesis (23)(a) and the definition of $\widetilde{\varphi}_{\eta}$, we know that $\widetilde{\varphi}_{\eta}$ is proper, convex and lower semicontinuous with $\operatorname{dom} \widetilde{\varphi}_{\eta}=K$. It is well known, cf., e.g., [9, Theorem 1.3.19] that the operator $T_{2}=\partial \widetilde{\varphi}_{\eta}: X \rightarrow 2^{X^{*}}$ is maximal monotone with $D\left(\partial \widetilde{\varphi}_{\eta}\right)=K$.

We are now in a position to apply a surjectivity result of Theorem 6 and deduce that there exists $u_{\eta} \in X$ a solution to inclusion (33). In what follows we observe that every solution to problem (33) is a solution to problem (32). Indeed, let $u_{\eta} \in X$ be such that

$$
A u_{\eta}+\xi_{\eta}+\theta_{\eta}=f
$$

with $\xi_{\eta} \in \partial \widetilde{\varphi}_{\eta}\left(u_{\eta}\right)$ and $\theta_{\eta} \in \partial j\left(u_{\eta}\right)$. We have

$$
\begin{aligned}
& \left\langle\xi_{\eta}, v-u_{\eta}\right\rangle \leq \widetilde{\varphi}_{\eta}(v)-\widetilde{\varphi}_{\eta}\left(u_{\eta}\right) \quad \text { for all } v \in X, \\
& \left\langle\theta_{\eta}, v\right\rangle \leq j^{0}\left(u_{\eta} ; v\right) \text { for all } v \in X .
\end{aligned}
$$

Combining (34) with the last two inequalities, we obtain

$$
\left\langle A u_{\eta}, v-u_{\eta}\right\rangle+\widetilde{\varphi}_{\eta}(v)-\widetilde{\varphi}_{\eta}\left(u_{\eta}\right)+j^{0}\left(u_{\eta} ; v-u_{\eta}\right) \geq\left\langle f, v-u_{\eta}\right\rangle \quad \text { for all } v \in X .
$$


This implies that $u_{\eta} \in X$ solves problem (32). We conclude that Problem 19 has at least one solution $u_{\eta} \in K$. i.e.,

For the uniqueness part, let $u_{1}, u_{2} \in K$ be solutions to Problem 19, for the same fixed $\eta$,

$$
\begin{array}{ll}
\left\langle A u_{1}, v-u_{1}\right\rangle+\varphi(\eta, v)-\varphi\left(\eta, u_{1}\right)+j^{0}\left(u_{1} ; v-u_{1}\right) \geq\left\langle f, v-u_{1}\right\rangle & \text { for all } v \in K \\
\left\langle A u_{2}, v-u_{2}\right\rangle+\varphi(\eta, v)-\varphi\left(\eta, u_{2}\right)+j^{0}\left(u_{2} ; v-u_{2}\right) \geq\left\langle f, v-u_{2}\right\rangle & \text { for all } v \in K
\end{array}
$$

Taking $v=u_{2}$ in the first inequality and $v=u_{1}$ in the second one, adding them, we obtain

$$
\left\langle A u_{1}-A u_{2}, u_{2}-u_{1}\right\rangle+j^{0}\left(u_{1} ; u_{2}-u_{1}\right)+j^{0}\left(u_{2} ; u_{1}-u_{2}\right) \geq 0 .
$$

From the strong monotonicity of $A$ and hypothesis (24)(c), we have

$$
\left(m_{A}-\alpha_{j}\right)\left\|u_{1}-u_{2}\right\|_{X}^{2} \leq 0
$$

which, due to the smallness condition (29), implies $u_{1}=u_{2}$. This completes the proof of the lemma.

Define now the operator $\Lambda: K \rightarrow K$ by

$$
\Lambda \eta=u_{\eta} \quad \text { for } \eta \in K
$$

where $u_{\eta} \in K$ denotes the unique solution of Problem 19.

Lemma 21 The operator $\Lambda$ has a unique fixed point.

Proof Let $\eta_{1}, \eta_{2} \in K$ and $u_{1}=u_{\eta_{1}}, u_{2}=u_{\eta_{2}} \in K$ be the unique solutions of Problem 19 corresponding to $\eta_{1}, \eta_{2}$, respectively. From the inequalities

$$
\begin{array}{ll}
\left\langle A u_{1}, v-u_{1}\right\rangle+\varphi\left(\eta_{1}, v\right)-\varphi\left(\eta_{1}, u_{1}\right)+j^{0}\left(u_{1} ; v-u_{1}\right) \geq\left\langle f, v-u_{1}\right\rangle & \text { for all } v \in K, \\
\left\langle A u_{2}, v-u_{2}\right\rangle+\varphi\left(\eta_{2}, v\right)-\varphi\left(\eta_{2}, u_{2}\right)+j^{0}\left(u_{2} ; v-u_{2}\right) \geq\left\langle f, v-u_{2}\right\rangle & \text { for all } v \in K,
\end{array}
$$

we have

$$
\begin{aligned}
\left\langle A u_{1}-A u_{2}, u_{1}-u_{2}\right\rangle \leq & \varphi\left(\eta_{1}, u_{2}\right)-\varphi\left(\eta_{1}, u_{1}\right)+\varphi\left(\eta_{2}, u_{1}\right)-\varphi\left(\eta_{2}, u_{2}\right) \\
& +j^{0}\left(u_{1} ; u_{2}-u_{1}\right)+j^{0}\left(u_{2} ; u_{1}-u_{2}\right) .
\end{aligned}
$$

We use the strong monotonicity of $A$, hypotheses (23)(b) and (24)(c), and obtain

$$
m_{A}\left\|u_{1}-u_{2}\right\|_{X}^{2} \leq \alpha_{\varphi}\left\|\eta_{1}-\eta_{2}\right\|_{X}\left\|u_{1}-u_{2}\right\|_{X}+\alpha_{j}\left\|u_{1}-u_{2}\right\|_{X}^{2} .
$$

Consequently,

$$
\left\|\Lambda \eta_{1}-\Lambda \eta_{2}\right\|_{X}=\left\|u_{1}-u_{2}\right\|_{X} \leq \frac{\alpha_{\varphi}}{m_{A}-\alpha_{j}}\left\|\eta_{1}-\eta_{2}\right\|_{X}
$$

From condition (29), by applying the Banach contraction principle, we deduce that there exists a unique $\eta^{*} \in K$ such that $\eta^{*}=\Lambda \eta^{*}$. This completes the proof of the lemma. 
We now have all the ingredients to provide the proof of the main result in this section.

Proof of Theorem 18 For the existence, let $\eta^{*} \in K$ be the fixed point of the operator $\Lambda$. We write inequality (31) for $\eta=\eta^{*}$ and observe that $u_{\eta^{*}}=\Lambda \eta^{*}=\eta^{*}$. Hence, we conclude that the function $\eta^{*} \in K$ is a solution to Problem 11 .

The uniqueness of a solution to Problem 11 is proved directly. Let $u_{1}, u_{2} \in K$ be solutions, i.e.,

$$
\begin{array}{ll}
\left\langle A u_{1}, v-u_{1}\right\rangle+\varphi\left(u_{1}, v\right)-\varphi\left(u_{1}, u_{1}\right)+j^{0}\left(u_{1} ; v-u_{1}\right) \geq\left\langle f, v-u_{1}\right\rangle & \text { for all } v \in K, \\
\left\langle A u_{2}, v-u_{2}\right\rangle+\varphi\left(u_{2}, v\right)-\varphi\left(u_{2}, u_{2}\right)+j^{0}\left(u_{2} ; v-u_{2}\right) \geq\left\langle f, v-u_{2}\right\rangle & \text { for all } v \in K .
\end{array}
$$

From these inequalities, we obtain

$$
\begin{aligned}
\left\langle A u_{1}-A u_{2}, u_{1}-u_{2}\right\rangle \leq & \varphi\left(u_{1}, u_{2}\right)-\varphi\left(u_{1}, u_{1}\right)+\varphi\left(u_{2}, u_{1}\right)-\varphi\left(u_{2}, u_{2}\right) \\
& +j^{0}\left(u_{1} ; u_{2}-u_{1}\right)+j^{0}\left(u_{2} ; u_{1}-u_{2}\right) .
\end{aligned}
$$

Conditions (22)(c), (23)(b) and (24)(c) imply

$$
m_{A}\left\|u_{1}-u_{2}\right\|_{X}^{2} \leq \alpha_{\varphi}\left\|u_{1}-u_{2}\right\|_{X}^{2}+\alpha_{j}\left\|u_{1}-u_{2}\right\|_{X}^{2}
$$

from which, due to the smallness assumption (29), it follows that $u_{1}=u_{2}$. This completes the proof of the theorem.

\section{Continuous Dependence on the Data}

In this section we study the continuous dependence of the solution on the data. We assume the hypotheses of Theorem 18 and denote by $u \in X$ the unique solution to Problem 11, guaranteed by this theorem.

Let $\rho>0$ be a parameter. Consider the functions $\varphi_{\rho}, j_{\rho}$ and $f_{\rho}$ which satisfy hypotheses (23), (24) and (26) with constants $\alpha_{\varphi_{\rho}}$ and $\alpha_{j_{\rho}}$, respectively. Assume that

$$
\begin{aligned}
& \text { there exists } m_{0} \in \mathbb{R} \text { such that } \alpha_{\varphi_{\rho}}+\alpha_{j_{\rho}} \leq m_{0}<m_{A}, \quad \text { for all } \rho>0, \\
& \alpha_{j_{\rho}}<\alpha_{A} \text { for all } \rho>0,
\end{aligned}
$$

and consider the following version of Problem 11.

Problem 22 Find $u_{\rho} \in K$ such that

$$
\left\langle A u_{\rho}, v-u_{\rho}\right\rangle+\varphi_{\rho}\left(u_{\rho}, v\right)-\varphi_{\rho}\left(u_{\rho}, u_{\rho}\right)+j_{\rho}^{0}\left(u_{\rho} ; v-u_{\rho}\right) \geq\left\langle f_{\rho}, v-u_{\rho}\right\rangle \quad \text { for all } v \in K \text {. }
$$

Theorem 18 guarantees that Problem 22 has a unique solution $u_{\rho} \in X$, for each $\rho>0$. We now consider the following hypotheses.

$$
\left\{\begin{array}{l}
\text { There exists a function } G: \mathbb{R}_{+} \rightarrow \mathbb{R}_{+} \text {and } g \in \mathbb{R}_{+} \text {such that } \\
\varphi(\eta, v)-\varphi(\eta, \eta)-\varphi_{\rho}(\eta, v)+\varphi_{\rho}(\eta, \eta) \leq G(\rho)\left(\|\eta\|_{X}+g\right)\|\eta-v\|_{X} \\
\text { for all } \eta, v \in X, \rho>0 \text { and } \lim _{\rho \rightarrow 0} G(\rho)=0
\end{array}\right.
$$


( There exists a function $H: \mathbb{R}_{+} \rightarrow \mathbb{R}_{+}$and $h \in \mathbb{R}_{+}$such that $j^{0}(u ; v)-j_{\rho}^{0}(u ; v) \leq H(\rho)\left(\|u\|_{X}+h\right)\|u-v\|_{X}$ for all $u, v \in X, \rho>0$ and $\lim _{\rho \rightarrow 0} H(\rho)=0$.

$$
f_{\rho} \rightarrow f \quad \text { in } X^{*}, \text { as } \rho \rightarrow 0 .
$$

Our main result in this section is the following.

Theorem 23 Assume that (22)-(26), (29), (30), (35)-(39) hold. Then $u_{\rho} \rightarrow u$ in $X$ as $\rho \rightarrow 0$.

Proof Let $\rho>0$. We take $v=u_{\rho}$ in Problem 11 and $v=u$ in Problem 22, and obtain

$$
\begin{aligned}
& u \in K, \quad\left\langle A u, u_{\rho}-u\right\rangle+\varphi\left(u, u_{\rho}\right)-\varphi(u, u)+j^{0}\left(u ; u_{\rho}-u\right) \geq\left\langle f, u_{\rho}-u\right\rangle \\
& u_{\rho} \in K, \quad\left\langle A u_{\rho}, u-u_{\rho}\right\rangle+\varphi_{\rho}\left(u_{\rho}, u\right)-\varphi_{\rho}\left(u_{\rho}, u_{\rho}\right)+j_{\rho}^{0}\left(u_{\rho} ; u-u_{\rho}\right) \geq\left\langle f_{\rho}, u-u_{\rho}\right\rangle .
\end{aligned}
$$

Adding the two inequalities, we have

$$
\begin{aligned}
\left\langle A u_{\rho}-A u, u_{\rho}-u\right\rangle \leq & \varphi\left(u, u_{\rho}\right)-\varphi(u, u)+\varphi_{\rho}\left(u_{\rho}, u\right)-\varphi_{\rho}\left(u_{\rho}, u_{\rho}\right) \\
& +j^{0}\left(u ; u_{\rho}-u\right)+j_{\rho}^{0}\left(u_{\rho} ; u-u_{\rho}\right)+\left\langle f_{\rho}-f, u_{\rho}-u\right\rangle .
\end{aligned}
$$

Next, we write

$$
\begin{aligned}
& \varphi\left(u, u_{\rho}\right)-\varphi(u, u)+\varphi_{\rho}\left(u_{\rho}, u\right)-\varphi_{\rho}\left(u_{\rho}, u_{\rho}\right) \\
& =\varphi\left(u, u_{\rho}\right)-\varphi(u, u)-\varphi_{\rho}\left(u, u_{\rho}\right)+\varphi_{\rho}(u, u) \\
& \quad+\varphi_{\rho}\left(u, u_{\rho}\right)-\varphi_{\rho}(u, u)+\varphi_{\rho}\left(u_{\rho}, u\right)-\varphi_{\rho}\left(u_{\rho}, u_{\rho}\right)
\end{aligned}
$$

and, using assumption (37) and condition (23)(c), we find that

$$
\begin{aligned}
& \varphi\left(u, u_{\rho}\right)-\varphi(u, u)+\varphi_{\rho}\left(u_{\rho}, u\right)-\varphi_{\rho}\left(u_{\rho}, u_{\rho}\right) \\
& \quad \leq G(\rho)\left(\|u\|_{X}+g\right)\left\|u_{\rho}-u\right\|_{X}+\alpha_{\varphi_{\rho}}\left\|u_{\rho}-u\right\|_{X}^{2} .
\end{aligned}
$$

In a similar way, writing

$$
\begin{aligned}
& j^{0}\left(u ; u_{\rho}-u\right)+j_{\rho}^{0}\left(u_{\rho} ; u-u_{\rho}\right) \\
& \quad=j^{0}\left(u ; u_{\rho}-u\right)-j_{\rho}^{0}\left(u ; u_{\rho}-u\right)+j_{\rho}^{0}\left(u ; u_{\rho}-u\right)+j_{\rho}^{0}\left(u_{\rho} ; u-u_{\rho}\right)
\end{aligned}
$$

and, using assumption (38) and condition (24)(c), we infer that

$$
\begin{aligned}
& j^{0}\left(u ; u_{\rho}-u\right)+j_{\rho}^{0}\left(u_{\rho} ; u-u_{\rho}\right) \\
& \quad \leq H(\rho)\left(\|u\|_{X}+h\right)\left\|u_{\rho}-u\right\|_{X}+\alpha_{j_{\rho}}\left\|u_{\rho}-u\right\|_{X}^{2} .
\end{aligned}
$$

Next, we use estimates (40)-(42), conditions (35), (36) and (22)(c) to find that

$$
\left(m_{A}-m_{0}\right)\left\|u_{\rho}-u\right\|_{X} \leq G(\rho)\left(\|u\|_{X}+g\right)+H(\rho)\left(\|u\|_{X}+h\right)+\left\|f_{\rho}-f\right\|_{X^{*}} .
$$

Taking into account (37)-(39), we deduce that $u_{\rho} \rightarrow u$ in $X$, as $\rho \rightarrow 0$ which concludes the proof. 


\section{A Penalty Method}

In this section we will prove the existence and uniqueness of solution to the variationalhemivariational inequality by applying a penalty method. We consider the following problem.

Problem 24 Find an element $u \in K$ such that

$$
\langle A u, v-u\rangle+\varphi(v)-\varphi(u)+j^{0}(u ; v-u) \geq\langle f, v-u\rangle \quad \text { for all } v \in K .
$$

Note that Problem 24 is a particular case of Problem 11 obtained for the function $\varphi$ independent of the first variable. We need the following additional hypotheses:

$$
\varphi: X \rightarrow \mathbb{R} \text { is convex and lower semicontinuous, }
$$

$$
\left\{\begin{array}{l}
j: X \rightarrow \mathbb{R} \text { is such that } \lim \sup j^{0}\left(u_{n} ; v-u_{n}\right) \leq j^{0}(u ; v-u) \\
\quad \text { for all } v \in X \text { and } u_{n} \rightarrow u \text { weakly in } X .
\end{array}\right.
$$

We adopt the following notion of the penalty operator, see [27].

Definition 25 A single-valued operator $P: X \rightarrow X^{*}$ is said to be a penalty operator of $K$ if $P$ is bounded, demicontinuous, monotone and $K=\left\{x \in X \mid P x=0_{X^{*}}\right\}$.

The next result provides an example of a penalty operator. Recall that any reflexive Banach space $X$ can be always considered as equivalently renormed strictly convex space and, therefore, the duality map $J: X \rightarrow 2^{X^{*}}$, defined by

$$
J x=\left\{x^{*} \in X^{*} \mid\left\langle x^{*}, x\right\rangle=\|x\|_{X}^{2}=\left\|x^{*}\right\|_{X^{*}}^{2}\right\} \text { for all } x \in X
$$

is a single-valued operator. Details can be found in [9, Proposition 1.3.27] and [33, Proposition 32.22].

Lemma 26 Let $X$ be a reflexive Banach space, $J: X \rightarrow X^{*}$ be the duality mapping and $P_{K}: X \rightarrow X$ be the projection operator on $K$. Then the mapping $P=J\left(I-P_{K}\right)$ (I denotes the identity map on $X$ ) is a penalty operator of $K$.

Assume in what follows that $P: X \rightarrow X^{*}$. Then, for every $\lambda>0$, we consider the following penalized problem.

Problem 27 Find an element $u_{\lambda} \in X$ such that

$$
\left\langle A u_{\lambda}, v-u_{\lambda}\right\rangle+\frac{1}{\lambda}\left\langle P u_{\lambda}, v-u_{\lambda}\right\rangle+\varphi(v)-\varphi\left(u_{\lambda}\right)+j^{0}\left(u_{\lambda} ; v-u_{\lambda}\right) \geq\left\langle f, v-u_{\lambda}\right\rangle
$$

for all $v \in X$, where $P: X \rightarrow X^{*}$ is the penalty operator of $K$.

Our main result of this section is the following.

Theorem 28 Assume (22), (24)-(26), (43), (44), $P$ is a penalty operator of $K$ and

$$
\alpha_{j}<\min \left\{\alpha_{A}, m_{A}\right\} .
$$

Then 
(i) for each $\lambda>0$, there exists a unique solution $u_{\lambda} \in X$ to Problem 27;

(ii) $u_{\lambda} \rightarrow u$ in $X$, as $\lambda \rightarrow 0$, where $u \in K$ is a unique solution to Problem 24.

Proof We start with the proof of (i). It is obvious that under hypothesis (43), the function $\bar{\varphi}: X \times X \rightarrow \mathbb{R}$ defined by $\bar{\varphi}(\eta, v)=\varphi(v)$ for $\eta, v \in X$ satisfies condition (23) with $\alpha_{\varphi}=0$. Using the properties of the penalty operator stated in Definition 25 and the fact that $D(P)=$ $X$, it follows (see Exercise I.9 in Sect. 1.9 of [9]) that $P$ is bounded, hemicontinuous and monotone. From Proposition 27.6 of [33], we deduce that $P$ is a pseudomotone operator. Now, we consider the operator $A_{\lambda}: X \rightarrow X^{*}$ defined by $A_{\lambda}=A+\frac{1}{\lambda} P$ for $\lambda>0$. From hypothesis (22), it is clear that $A_{\lambda}$ is pseudomonotone, $u_{0}$-coercive and strongly monotone. Therefore, by applying Theorem 18, we deduce that for each $\lambda>0$ there exists a unique solution $u_{\lambda} \in X$ to Problem 27.

Next, we pass to the proof of (ii). We claim that there are an element $\tilde{u} \in X$ and a subsequence of $u_{\lambda}$, denoted in the same way, such that $u_{\lambda} \rightarrow \tilde{u}$ weakly in $X$, as $\lambda \rightarrow 0$. To this end, we will establish the boundedness of $\left\{u_{\lambda}\right\}$ in $X$. First, by hypothesis (24), we have

$$
\begin{aligned}
j^{0}\left(u_{\lambda} ; u_{0}-u_{\lambda}\right) & =j^{0}\left(u_{\lambda} ; u_{0}-u_{\lambda}\right)+j^{0}\left(u_{0} ; u_{\lambda}-u_{0}\right)-j^{0}\left(u_{0} ; u_{\lambda}-u_{0}\right) \\
& \leq \alpha_{j}\left\|u_{\lambda}-u_{0}\right\|_{X}^{2}+\left|\max \left\{\left\langle\zeta, u_{\lambda}-u_{0}\right\rangle \mid \zeta \in \partial j\left(u_{0}\right)\right\}\right| \\
& \leq \alpha_{j}\left\|u_{\lambda}-u_{0}\right\|_{X}^{2}+\left(c_{0}+c_{1}\left\|u_{0}\right\|_{X}\right)\left\|u_{\lambda}-u_{0}\right\|_{X} .
\end{aligned}
$$

Subsequently, since $\varphi$ is convex and lower semicontinuous, it admits an affine minorant, cf., e.g., Proposition 5.2.25 in [8], that is, there are $l \in X^{*}$ and $b \in \mathbb{R}$ such that

$$
\varphi(v) \geq\langle l, v\rangle+b \quad \text { for all } v \in X .
$$

We now put $v=u_{0} \in K$ in Problem 27, use (47), (48) and the strong monotonicity of the operator $A$, and obtain

$$
\begin{aligned}
m_{A}\left\|u_{\lambda}-u_{0}\right\|_{X}^{2} \leq & \left\langle A u_{\lambda}-A u_{0}, u_{\lambda}-u_{0}\right\rangle=\left\langle A u_{\lambda}, u_{\lambda}-u_{0}\right\rangle-\left\langle A u_{0}, u_{\lambda}-u_{0}\right\rangle \\
\leq & \frac{1}{\lambda}\left\langle P u_{\lambda}, u_{0}-u_{\lambda}\right\rangle+\varphi\left(u_{0}\right)-\varphi\left(u_{\lambda}\right)+j^{0}\left(u_{\lambda} ; u_{0}-u_{\lambda}\right) \\
& +\left\langle f-A u_{0}, u_{\lambda}-u_{0}\right\rangle \\
\leq & -\frac{1}{\lambda}\left\langle P u_{0}-P u_{\lambda}, u_{0}-u_{\lambda}\right\rangle+\varphi\left(u_{0}\right)-\left\langle l, u_{\lambda}\right\rangle-b+\alpha_{j}\left\|u_{\lambda}-u_{0}\right\|_{X}^{2} \\
& +\left(c_{0}+c_{1}\left\|u_{0}\right\|_{X}\right)\left\|u_{\lambda}-u_{0}\right\|_{X}+\left\|f-A u_{0}\right\|_{X^{*}}\left\|u_{\lambda}-u_{0}\right\|_{X} .
\end{aligned}
$$

Hence, by the monotonicity of $P$, we have

$$
\begin{aligned}
\left(m_{A}-\alpha_{j}\right)\left\|u_{\lambda}-u_{0}\right\|_{X}^{2} \leq & \left|\varphi\left(u_{0}\right)\right|+\|l\|_{X^{*}}\left\|u_{\lambda}\right\|_{X}+|b| \\
& +\left\|u_{\lambda}-u_{0}\right\|_{X}\left(c_{0}+c_{1}\left\|u_{0}\right\|_{X}+\left\|f-A u_{0}\right\|_{X^{*}}\right),
\end{aligned}
$$

which, due to (46), implies that there is a constant $C>0$ independent of $\lambda$ such that $\left\|u_{\lambda}\right\|_{X} \leq C$. Thus, from the reflexivity of $X$, we deduce, by passing to a subsequence, if necessary, that

$$
u_{\lambda} \rightarrow \tilde{u} \quad \text { weakly in } X, \text { as } \lambda \rightarrow 0
$$

with some $\tilde{u} \in X$. This implies the claim. 
Next, we show that $\tilde{u} \in K$ is a solution to Problem 24. From (45), exploiting hypotheses (22), (24) and property (48), we obtain

$$
\begin{aligned}
\frac{1}{\lambda}\left\langle P u_{\lambda}, u_{\lambda}-v\right\rangle \leq & \left\langle A u_{\lambda}, v-u_{\lambda}\right\rangle+\varphi(v)-\varphi\left(u_{\lambda}\right)+j^{0}\left(u_{\lambda} ; v-u_{\lambda}\right)+\left\langle f, u_{\lambda}-v\right\rangle \\
\leq & -\left\langle A u_{\lambda}-A v, u_{\lambda}-v\right\rangle-\left\langle A v, u_{\lambda}-v\right\rangle+\varphi(v)-\varphi\left(u_{\lambda}\right) \\
& +\left(c_{0}+c_{1}\left\|u_{\lambda}\right\|_{X}\right)\left\|u_{\lambda}-v\right\|_{X}+\left\langle f, u_{\lambda}-v\right\rangle \\
\leq & \left(\|A v-f\|_{X^{*}}+c_{0}+c_{1}\left\|u_{\lambda}\right\|_{X}\right)\left\|u_{\lambda}-v\right\|_{X}+\varphi(v)-\varphi\left(u_{\lambda}\right) \\
\leq & \left(\|A v-f\|_{X^{*}}+c_{0}+c_{1}\left\|u_{\lambda}\right\|_{X}\right)\left\|u_{\lambda}-v\right\|_{X}+\|l\|_{X^{*}}\left\|u_{\lambda}\right\|_{X}+|b|+\varphi(v)
\end{aligned}
$$

for all $v \in X$. Since $\left\|u_{\lambda}\right\|_{X} \leq C$, we infer that

$$
\frac{1}{\lambda}\left\langle P u_{\lambda}, u_{\lambda}-v\right\rangle \leq c_{v} \quad \text { for all } v \in X
$$

where $c_{v}$ depends on $v$ and is independent of $\lambda$. Choosing $v=\widetilde{u}$ in (50), we have

$$
\limsup _{\lambda \rightarrow 0}\left\langle P u_{\lambda}, u_{\lambda}-\widetilde{u}\right\rangle \leq 0 .
$$

Exploiting the pseudomonotonicty of $P$, cf. Definition 9, from (49) and (51), we have

$$
\langle P \tilde{u}, \tilde{u}-v\rangle \leq \liminf _{\lambda \rightarrow 0}\left\langle P u_{\lambda}, u_{\lambda}-v\right\rangle \quad \text { for all } v \in X .
$$

From (51) and (52), we obtain $\langle P \tilde{u}, \tilde{u}-v\rangle \leq 0$ for all $v \in X$. Hence, choosing $v=\tilde{u}+w$ with $w \in X$, we get $\langle P \widetilde{u}, w\rangle=0$ for all $w \in X$. So, it is clear that $P \widetilde{u}=0$ which means that $\tilde{u} \in K$.

Subsequently, testing (45) by $v \in K$ and using the monotonicity of $P$, we have

$$
\left\langle A u_{\lambda}, u_{\lambda}-v\right\rangle \leq-\frac{1}{\lambda}\left\langle P v-P u_{\lambda}, v-u_{\lambda}\right\rangle+\varphi(v)-\varphi\left(u_{\lambda}\right)+j^{0}\left(u_{\lambda} ; v-u_{\lambda}\right)+\left\langle f, u_{\lambda}-v\right\rangle,
$$

which implies that

$$
\left\langle A u_{\lambda}, u_{\lambda}-v\right\rangle \leq \varphi(v)-\varphi\left(u_{\lambda}\right)+j^{0}\left(u_{\lambda} ; v-u_{\lambda}\right)+\left\langle f, u_{\lambda}-v\right\rangle
$$

for all $v \in K$. Using (49) and weak lower semicontinuity of $\varphi$ (recall that this follows from hypothesis (43)), we have

$$
\limsup _{\lambda \rightarrow 0}\left(\varphi(\widetilde{u})-\varphi\left(u_{\lambda}\right)\right) \leq 0 .
$$

On the other hand, from hypothesis (44) and (49), it follows that

$$
\limsup _{\lambda \rightarrow 0} j^{0}\left(u_{\lambda} ; \tilde{u}-u_{\lambda}\right) \leq 0 .
$$

Now, taking $v=\tilde{u} \in K$ in (53) and using (49), (54) and (55), we obtain

$$
\limsup _{\lambda \rightarrow 0}\left\langle A u_{\lambda}, u_{\lambda}-\widetilde{u}\right\rangle \leq 0 .
$$


This inequality together with (49) and the pseudomonotonicity of $A$ implies

$$
\langle A \tilde{u}, \tilde{u}-v\rangle \leq \liminf _{\lambda \rightarrow 0}\left\langle A u_{\lambda}, u_{\lambda}-v\right\rangle \quad \text { for all } v \in X .
$$

We are now in a position to pass to the upper limit in (53). Using (49), weak lower semicontinuity of $\varphi$ and (44), we obtain

$$
\limsup _{\lambda \rightarrow 0}\left\langle A u_{\lambda}, u_{\lambda}-v\right\rangle \leq \varphi(v)-\varphi(\widetilde{u})+j^{0}(\tilde{u} ; v-\widetilde{u})+\langle f, \tilde{u}-v\rangle,
$$

for all $v \in K$. Combining (56) and (57), we have

$$
\langle A \tilde{u}, \tilde{u}-v\rangle \leq \varphi(v)-\varphi(\tilde{u})+j^{0}(\tilde{u} ; v-\tilde{u})+\langle f, \tilde{u}-v\rangle,
$$

for all $v \in K$. Hence, it follows that $\tilde{u} \in K$ is a solution to Problem 24 .

Since Problem 24 has a unique solution $u \in K$, we deduce that $\tilde{u}=u$. This implies that every subsequence of $\left\{u_{\lambda}\right\}$ which converges weakly has the same limit and, therefore, it follows that the whole sequence $\left\{u_{\lambda}\right\}$ converges weakly to $u$.

In the final step of the proof, we prove that $u_{\lambda} \rightarrow u$ in $X$, as $\lambda \rightarrow 0$. We take $v=\tilde{u} \in K$ in both (56) and (57) to obtain

$$
0 \leq \liminf _{\lambda \rightarrow 0}\left\langle A u_{\lambda}, u_{\lambda}-\widetilde{u}\right\rangle \quad \text { and } \quad \limsup _{\lambda \rightarrow 0}\left\langle A u_{\lambda}, u_{\lambda}-\widetilde{u}\right\rangle \leq 0
$$

respectively, which gives $\left\langle A u_{\lambda}, u_{\lambda}-\tilde{u}\right\rangle \rightarrow 0$, as $\lambda \rightarrow 0$. Therefore, using the strong monotonicity of $A$ and the convergence $u_{\lambda} \rightarrow u$ weakly in $X$, we have

$$
m_{A}\left\|u_{\lambda}-u\right\|_{X}^{2} \leq\left\langle A u_{\lambda}-A u, u_{\lambda}-u\right\rangle=\left\langle A u_{\lambda}, u_{\lambda}-u\right\rangle-\left\langle A u, u_{\lambda}-u\right\rangle \rightarrow 0,
$$

as $\lambda \rightarrow 0$. It follows from here that $u_{\lambda} \rightarrow u$ in $X$ which completes the proof of the theorem.

We end this section with the following result, which provides sufficient conditions for functions which satisfy our hypotheses (24) and (44).

Lemma 29 Let $X$ and $Y$ be reflexive Banach spaces, $\psi: Y \rightarrow \mathbb{R}$ be a function which satisfies (24) and $\psi($ or $-\psi)$ is regular, and let $M: X \rightarrow Y$ be given by $M v=L v+v_{0}$, where $L: X \rightarrow Y$ is a linear compact operator and $v_{0} \in Y$ is fixed. Define the function $j: X \rightarrow \mathbb{R}$ by $j(v)=\psi(M v)$ for $v \in X$. Then the function $j$ satisfies conditions (24) and (44).

Proof From the chain rule for the Clarke subgradient, cf. Proposition 3.37 of [21], it is clear that $j$ is locally Lipschitz and

$$
\begin{aligned}
& \text { (a) } \partial j(u) \subset L^{*} \partial \psi(M u) \quad \text { for all } u \in X, \\
& \text { (b) } j^{0}(u ; v) \leq \psi^{0}(M u ; L v) \quad \text { for all } u, v \in X \text {, }
\end{aligned}
$$

and if $\psi($ or $-\psi)$ is regular, then $j$ (or $-j$ ) is regular and (a) and (b) hold with equalities. Using (a) and condition (24)(b) for $\psi$, we have

$$
\begin{aligned}
\|\partial j(v)\|_{X^{*}} & \leq\left\|L^{*}\right\|\|\partial \psi(M v)\|_{Y^{*}} \leq\left\|L^{*}\right\|\left(c_{0}+c_{1}\|M v\|_{Y}\right) \\
& \leq c_{0}\left\|L^{*}\right\|+c_{1}\left\|L^{*}\right\|\left(\|L v\|_{Y}+\left\|v_{0}\right\|_{Y}\right) \leq \widetilde{c}_{0}+\widetilde{c}_{1}\|v\|_{X}
\end{aligned}
$$


for all $v \in X$ with $\widetilde{c}_{0}=c_{0}\left\|L^{*}\right\|+c_{1}\|L\|\left\|v_{0}\right\|_{Y}$ and $\widetilde{c}_{1}=c_{1}\|L\|^{2}$, where $\|L\|=\|L\|_{\mathcal{L}(X, Y)}$ and $\left\|L^{*}\right\|=\left\|L^{*}\right\|_{\mathcal{L}\left(Y^{*}, X^{*}\right)}$. This means that $j$ satisfies condition (24)(b).

Next, using (b) and condition (24)(c) for $\psi$, we obtain

$$
\begin{aligned}
& j^{0}\left(v_{1} ; v_{2}-v_{1}\right)+j^{0}\left(v_{2} ; v_{1}-v_{2}\right) \\
& \quad \leq \psi^{0}\left(M v_{1} ; L\left(v_{2}-v_{1}\right)\right)+\psi^{0}\left(M v_{2} ; L\left(v_{1}-v_{2}\right)\right) \\
& \quad \leq \alpha_{\psi}\|L\|^{2}\left\|v_{1}-v_{2}\right\|_{X}^{2}
\end{aligned}
$$

for all $v_{1}, v_{2} \in X$. Therefore, $j$ satisfies (24)(c) with $\alpha_{j}=\alpha_{\psi}\|L\|^{2}$. We conclude from above that $j$ satisfies condition (24).

In order to establish condition (44) for $j$, we suppose that $u_{n} \rightarrow u$ weakly in $X$ and $v \in X$. From the compactness of the operator $L: X \rightarrow Y$, we have $M u_{n}=L u_{n}+v_{0} \rightarrow$ $L u+v_{0}=M u$ in $Y$. Exploiting the upper semicontinuity of $\psi^{0}(\cdot ; \cdot)$, cf. Proposition 3.23(ii) of [21], it follows that

$$
\begin{aligned}
\limsup j^{0}\left(u_{n} ; v-u_{n}\right) & \leq \limsup \psi^{0}\left(M u_{n} ; L\left(v-u_{n}\right)\right) \\
& \leq \psi^{0}(M u ; L(v-u))=j^{0}(u ; v-u) .
\end{aligned}
$$

Note that the last equality follows from the regularity of $\psi$ (or $-\psi$ ). Thus, the function $j$ satisfies (44) which concludes the proof.

\section{A Contact Problem with Unilateral Constraints}

In this section we introduce and study an elastic contact problem for which the results of Sects 4-6 can be applied. The classical formulation of the problem is the following:

Problem 30 Find a displacement field $\boldsymbol{u}: \Omega \rightarrow \mathbb{R}^{d}$, a stress field $\sigma: \Omega \rightarrow \mathbb{S}^{d}$ and an interface force $\xi_{v}: \Gamma_{3} \rightarrow \mathbb{R}$ such that

$$
\begin{aligned}
& \boldsymbol{\sigma}=\mathcal{F} \boldsymbol{\varepsilon}(\boldsymbol{u}) \quad \text { in } \Omega, \\
& \operatorname{Div} \boldsymbol{\sigma}+\boldsymbol{f}_{0}=\mathbf{0} \quad \text { in } \Omega, \\
& \boldsymbol{u}=\mathbf{0} \quad \text { on } \Gamma_{1}, \\
& \boldsymbol{\sigma} \boldsymbol{v}=\boldsymbol{f}_{2} \quad \text { on } \Gamma_{2}, \\
& u_{v} \leq g, \quad \sigma_{v}+\xi_{v} \leq 0, \quad\left(u_{v}-g\right)\left(\sigma_{v}+\xi_{v}\right)=0, \quad \xi_{v} \in \partial j_{v}\left(u_{v}\right) \quad \text { on } \Gamma_{3}, \\
& \left\|\boldsymbol{\sigma}_{\tau}\right\| \leq F_{b}\left(u_{v}\right), \quad-\boldsymbol{\sigma}_{\tau}=F_{b}\left(u_{v}\right) \frac{\boldsymbol{u}_{\tau}}{\left\|\boldsymbol{u}_{\tau}\right\|} \quad \text { if } \boldsymbol{u}_{\tau} \neq \mathbf{0} \quad \text { on } \Gamma_{3} .
\end{aligned}
$$

Here and below $\Omega$ represents the reference configuration of the elastic body and is assumed to be an open, bounded, connected set in $\mathbb{R}^{d}(d=2,3)$ with a Lipschitz boundary $\partial \Omega=\Gamma$. The set $\Gamma$ is partitioned into three disjoint and measurable parts $\Gamma_{1}, \Gamma_{2}$ and $\Gamma_{3}$ such that meas $\left(\Gamma_{1}\right)$ is positive. We use the notation $\boldsymbol{x}=\left(x_{i}\right)$ for a generic point in $\Omega \cup \Gamma$ and $\boldsymbol{v}=\left(v_{i}\right)$ for the outward unit normal at $\Gamma$. The indices $i, j, k, l$ run between 1 and $d$ and, unless stated otherwise, the summation convention over repeated indices is used. Notation $\mathbb{S}^{d}$ stands for the space of second order symmetric tensors on $\mathbb{R}^{d}$. On the spaces $\mathbb{R}^{d}$ and 
$\mathbb{S}^{d}$ we use the inner products and the Euclidean norms defined by

$$
\begin{aligned}
& \boldsymbol{u} \cdot \boldsymbol{v}=u_{i} v_{i}, \quad\|\boldsymbol{v}\|=(\boldsymbol{v} \cdot \boldsymbol{v})^{1 / 2} \quad \text { for all } \boldsymbol{u}=\left(u_{i}\right), \boldsymbol{v}=\left(v_{i}\right) \in \mathbb{R}^{d}, \\
& \boldsymbol{\sigma} \cdot \boldsymbol{\tau}=\sigma_{i j} \tau_{i j}, \quad\|\boldsymbol{\tau}\|=(\boldsymbol{\tau} \cdot \boldsymbol{\tau})^{1 / 2} \quad \text { for all } \boldsymbol{\sigma}=\left(\sigma_{i j}\right), \boldsymbol{\tau}=\left(\tau_{i j}\right) \in \mathbb{S}^{d},
\end{aligned}
$$

respectively. For a vector field, notation $v_{v}$ and $\boldsymbol{v}_{\tau}$ represent the normal and tangential components of $\boldsymbol{v}$ on $\Gamma$ given by $v_{\nu}=\boldsymbol{v} \cdot \boldsymbol{v}$ and $\boldsymbol{v}_{\tau}=\boldsymbol{v}-v_{\nu} \boldsymbol{v}$. Also, $\sigma_{v}$ and $\boldsymbol{\sigma}_{\tau}$ represent the normal and tangential components of the stress field $\sigma$ on the boundary, i.e., $\sigma_{v}=(\sigma \boldsymbol{v}) \cdot \boldsymbol{v}$ and $\boldsymbol{\sigma}_{\tau}=\boldsymbol{\sigma} \boldsymbol{v}-\sigma_{\nu} \boldsymbol{v}$.

A description of the equations and conditions in Problem 30 is the following. First, equation (58) is the constitutive law for elastic materials in which $\mathcal{F}$ represents the elasticity operator and $\boldsymbol{\varepsilon}(\boldsymbol{u})$ denotes the linearized strain tensor. Equation (59) is the equilibrium equation in which $f_{0}$ represents the density of the body forces. We use it here since we assume that the process is static and, therefore, we neglect the inertial term in the equation of motion. Conditions (60) and (61) represent the classical displacement-traction boundary conditions. They show that the body is fixed on $\Gamma_{1}$ and surface tractions of density $\boldsymbol{f}_{2}$ act on $\Gamma_{2}$. Relations (62) and (63), formulated on the surface $\Gamma_{3}$, represent the contact and the friction law, respectively. Here $g>0, \partial j_{\nu}$ denotes the Clarke subdifferential of the given function $j_{v}$, and $F_{b}$ denotes a positive function, the friction bound. More details and mechanical interpretation on static contact models with elastic materials could be found in the book [21].

Note that condition (62) models the contact with a foundation made of a rigid body covered by a layer made of elastic material, say asperities. It shows that the penetration is restricted, since $u_{v} \leq g$, where $g$ represents the thickness of the elastic layer. Also, when there is penetration, as far as the normal displacement does not reach the bound $g$, the contact is described with a multivalued normal compliance condition since, in this case, $-\sigma_{v}=\xi_{v} \in$ $\partial j_{v}\left(u_{v}\right)$. It follows from here that the unknown $\xi_{v}$ could be interpreted as the opposite of the normal stress on the contact surface. The friction law (63) has already been used in [30], associated to a multivalued normal compliance contact condition without unilateral constraint. Note that the friction bound $F_{b}$ is assumed to depend on the normal displacement $u_{v}$, which is reasonable from the physical point of view, as explained in [30]. Considering the friction law (63) associated to the normal compliance contact condition with unilateral constraints (62) represents the novelty of our contact model.

In the rest of the paper we use standard notation for Lebesgue and Sobolev spaces and, in addition, we use the spaces $V$ and $\mathcal{H}$ defined by

$$
\begin{aligned}
& V=\left\{\boldsymbol{v}=\left(v_{i}\right) \in H^{1}\left(\Omega ; \mathbb{R}^{d}\right) \mid \boldsymbol{v}=\mathbf{0} \text { a.e. on } \Gamma_{1}\right\}, \\
& \mathcal{H}=\left\{\boldsymbol{\tau}=\left(\tau_{i j}\right) \in L^{2}\left(\Omega ; \mathbb{S}^{d}\right)\right\} .
\end{aligned}
$$

Here and below we still denote by $\boldsymbol{v}$ the trace of an element $\boldsymbol{v} \in H^{1}\left(\Omega ; \mathbb{R}^{d}\right)$. The space $\mathcal{H}$ will be endowed with the Hilbertian structure given by the inner product

$$
(\boldsymbol{\sigma}, \boldsymbol{\tau})_{\mathcal{H}}=\int_{\Omega} \sigma_{i j}(\boldsymbol{x}) \tau_{i j}(\boldsymbol{x}) d x, \quad \sigma, \tau \in \mathcal{H}
$$

and the associated norm $\|\cdot\|_{\mathcal{H}}$. On the space $V$ we consider the inner product

$$
(\boldsymbol{u}, \boldsymbol{v})_{V}=(\boldsymbol{\varepsilon}(\boldsymbol{u}), \boldsymbol{\varepsilon}(\boldsymbol{v}))_{\mathcal{H}}, \quad \boldsymbol{u}, \boldsymbol{v} \in V
$$


and the associated norm $\|\cdot\|_{V}$. Recall that, since meas $\left(\Gamma_{1}\right)>0$, it follows that $V$ is a real Hilbert space. Moreover, by the Sobolev trace theorem, we have

$$
\|\boldsymbol{v}\|_{L^{2}\left(\Gamma_{3} ; \mathbb{R}^{d}\right)} \leq c_{k}\|\gamma\|\|\boldsymbol{v}\|_{V} \quad \text { for all } \boldsymbol{v} \in V
$$

$c_{k}>0$ being a constant in the Korn inequality and $\|\gamma\|$ being the norm of the trace operator $\gamma: V \rightarrow L^{2}\left(\Gamma_{3} ; \mathbb{R}^{d}\right)$.

In the study of Problem 30 we assume that the elasticity operator $\mathcal{F}$ satisfies the following condition.

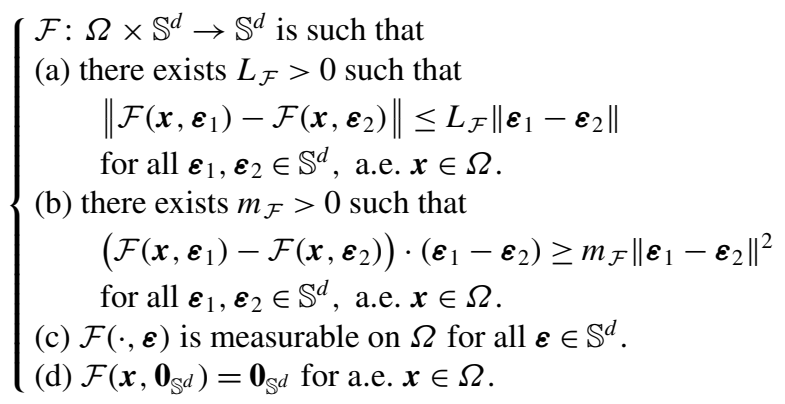

In addition, the friction bound $F_{b}$ and the potential function $j_{v}$ are such that

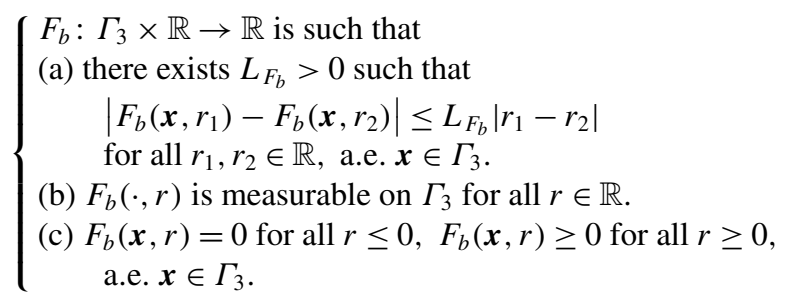

$j_{v}: \Gamma_{3} \times \mathbb{R} \rightarrow \mathbb{R}$ is such that

(a) $j_{v}(\cdot, r)$ is measurable on $\Gamma_{3}$ for all $r \in \mathbb{R}$ and there exists $\bar{e} \in L^{2}\left(\Gamma_{3}\right)$ such that $j_{v}(\cdot, \bar{e}(\cdot)) \in L^{1}\left(\Gamma_{3}\right)$.

(b) $j_{v}(\boldsymbol{x}, \cdot)$ is locally Lipschitz on $\mathbb{R}$ for a.e. $\boldsymbol{x} \in \Gamma_{3}$.

(c) $\left|\partial j_{v}(\boldsymbol{x}, r)\right| \leq \bar{c}_{0}+\bar{c}_{1}|r|$ for a.e. $\boldsymbol{x} \in \Gamma_{3}$,

(d) $j_{v}^{0}\left(\boldsymbol{x}, r_{1} ; r_{2}-r_{1}\right)+j_{v}^{0}\left(\boldsymbol{x}, r_{2} ; r_{1}-r_{2}\right) \leq \alpha_{j_{v}}\left|r_{1}-r_{2}\right|^{2}$ for a.e. $\boldsymbol{x} \in \Gamma_{3}$, all $r_{1}, r_{2} \in \mathbb{R}$ with $\alpha_{j_{v}} \geq 0$.

Finally, we assume that the densities of body forces and surface tractions have the regularity

$$
\boldsymbol{f}_{0} \in L^{2}\left(\Omega ; \mathbb{R}^{d}\right), \quad \boldsymbol{f}_{2} \in L^{2}\left(\Gamma_{2} ; \mathbb{R}^{d}\right) .
$$

Next, we introduce the set of admissible displacement fields $U$, and the element $f \in V^{*}$ defined by

$$
\begin{aligned}
& U=\left\{\boldsymbol{v} \in V \mid v_{v} \leq g \text { a.e. on } \Gamma_{3}\right\}, \\
& \langle\boldsymbol{f}, \boldsymbol{v}\rangle=\left(\boldsymbol{f}_{0}, \boldsymbol{v}\right)_{L^{2}\left(\Omega ; \mathbb{R}^{d}\right)}+\left(\boldsymbol{f}_{2}, \boldsymbol{v}\right)_{L^{2}\left(\Gamma_{2} ; \mathbb{R}^{d}\right)} \quad \text { for all } \boldsymbol{v} \in V .
\end{aligned}
$$


Then, using integration by parts and standard arguments we derive the following variational formulation for Problem 30.

Problem 31 Find a displacement field $\boldsymbol{u} \in U$ such that

$$
\begin{aligned}
& (\mathcal{F} \boldsymbol{\varepsilon}(\boldsymbol{u}), \boldsymbol{\varepsilon}(\boldsymbol{v})-\boldsymbol{\varepsilon}(\boldsymbol{u}))_{\mathcal{H}}+\int_{\Gamma_{3}} F_{b}\left(u_{v}\right)\left(\left\|\boldsymbol{v}_{\tau}\right\|-\left\|\boldsymbol{u}_{\tau}\right\|\right) d \Gamma \\
& \quad+\int_{\Gamma_{3}} j_{v}^{0}\left(u_{v} ; v_{v}-u_{v}\right) d \Gamma \geq\langle\boldsymbol{f}, \boldsymbol{v}-\boldsymbol{u}\rangle \quad \text { for all } \boldsymbol{v} \in U .
\end{aligned}
$$

We proceed with the following existence and uniqueness result.

Theorem 32 Assume (64)-(67) and, in addition, assume the smallness condition

$$
\left(L_{F_{b}}+\alpha_{j_{v}}\right) c_{k}^{2}\|\gamma\|^{2}<m_{\mathcal{F}}
$$

Then Problem 31 has a unique solution $\boldsymbol{u} \in U$.

Proof We shall apply Theorem 18 in the following functional framework: $X=V, K=U$,

$$
\begin{aligned}
& A: V \rightarrow V^{*}, \quad\langle A \boldsymbol{u}, \boldsymbol{v}\rangle=(\mathcal{F} \boldsymbol{\varepsilon}(\boldsymbol{u}), \boldsymbol{\varepsilon}(\boldsymbol{v}))_{\mathcal{H}} \quad \text { for } \boldsymbol{u}, \boldsymbol{v} \in V, \\
& \varphi: V \times V \rightarrow \mathbb{R}, \quad \varphi(\boldsymbol{u}, \boldsymbol{v})=\int_{\Gamma_{3}} F_{b}\left(\boldsymbol{x}, u_{v}(\boldsymbol{x})\right)\left\|\boldsymbol{v}_{\tau}(\boldsymbol{x})\right\| d \Gamma \quad \text { for } \boldsymbol{u}, \boldsymbol{v} \in V, \\
& j: V \rightarrow \mathbb{R}, \quad j(\boldsymbol{v})=\int_{\Gamma_{3}} j_{v}\left(\boldsymbol{x}, v_{v}(\boldsymbol{x})\right) d \Gamma \quad \text { for } \boldsymbol{v} \in V
\end{aligned}
$$

and $f \equiv f \in V^{*}$ defined by (69). To this end we verify that for $A$ defined by (71), hypothesis (64) implies (22) with $\alpha_{A}=m_{A}=m_{\mathcal{F}}$ (cf. [21], p. 205); for $\varphi$ defined by (72), (65) implies (23) with $\alpha_{\varphi}=L_{F_{b}} c_{k}^{2}\|\gamma\|^{2}$. Hypothesis (66)(a) guaranties that the function $j$, given by (73), is well defined, and conditions (24)(a) and (b) follow from (66)(b) and (c), respectively (see Theorem 3.47 in [21]), whereas property (24)(c) is a consequence of the inequality

$$
j^{0}(\boldsymbol{u} ; \boldsymbol{v}) \leq \int_{\Gamma_{3}} j_{v}^{0}\left(\boldsymbol{x}, u_{v}(\boldsymbol{x}) ; v_{v}(\boldsymbol{x})\right) d \Gamma \quad \text { for all } \boldsymbol{u}, \boldsymbol{v} \in V
$$

combined with the hypotheses (66)(d). Thus, (24) holds with $\alpha_{j}=\alpha_{j_{v}} c_{k}^{2}\|\gamma\|^{2}$. Moreover, it is easy to see that the set (68) is a nonempty, closed, convex set in $V$ and, therefore, condition (25) is satisfied. In addition, for $f$, assumption (67) implies (26). Next, considering the above relationships between constants, we immediately have that assumption (70) implies smallness conditions (29) and (30). Theorem 32 is now a direct consequence of Theorem 18.

Note that the unknowns of the contact problem 30 are the displacement field $\boldsymbol{u}$, the stress field $\sigma$ and the interface force $\xi_{v}$. In contrast, its variational formulation, Problem 31, is formulated only in terms of displacement. We conclude that Theorem 32 provides the unique weak solvability of Problem 30, in terms of displacement. However, once the displacement field is obtained by solving Problem 31, then the stress field $\sigma$ is uniquely determined by using the constitutive law (58). This implies the uniqueness of the solution to Problem 30, 
in $\boldsymbol{u}$ and $\boldsymbol{\sigma}$. Nevertheless, since the subdifferential is in general a multivalued operator, the inclusion $\xi_{v} \in \partial j_{v}\left(u_{v}\right)$ does not provide the uniqueness of $\xi_{v}$. Therefore, we are not able to provide the uniqueness of the contact interface force.

Next, we mention that Theorem 23 can be used to study the dependence of the weak solution of Problem 30 with respect to perturbations of the data and to prove its continuous dependence on the friction bound, the normal compliance function, and the densities of body forces and surface tractions. Such a result was already obtained in [30] in the study of a frictional contact problem without unilateral constraints and, therefore, we skip the details. Instead, we illustrate the use of the abstract result in Theorem 23 in the study of the frictionless version of Problem 30. To this end, we assume in what follows that friction bound vanishes, i.e.,

$$
F_{b} \equiv 0,
$$

and we consider a normal compliance function $p_{v}$ which satisfies

$$
\left\{\begin{array}{l}
p_{v}: \Gamma_{3} \times \mathbb{R} \rightarrow \mathbb{R}_{+} \text {is such that } \\
\text { (a) there exists } L_{p_{v}}>0 \text { such that } \\
\quad\left|p_{v}\left(\boldsymbol{x}, r_{1}\right)-p_{v}\left(\boldsymbol{x}, r_{2}\right)\right| \leq L_{p_{v}}\left|r_{1}-r_{2}\right| \\
\quad \text { for all } r_{1}, r_{2} \in \mathbb{R} \text {, a.e. } \boldsymbol{x} \in \Gamma_{3} . \\
\text { (b) }\left(p_{v}\left(\boldsymbol{x}, r_{1}\right)-p_{v}\left(\boldsymbol{x}, r_{2}\right)\right)\left(r_{1}-r_{2}\right) \geq 0 \\
\quad \text { for all } r_{1}, r_{2} \in \mathbb{R}, \text { a.e. } \boldsymbol{x} \in \Gamma_{3} . \\
\text { (c) } p_{v}(\boldsymbol{x}, r)=0 \text { for all } r \leq 0, \text { a.e. } \boldsymbol{x} \in \Gamma_{3} . \\
\text { (d) } p_{v}(\cdot, r) \text { is measurable on } \Gamma_{3} \text { for all } r \in \mathbb{R} \text {. }
\end{array}\right.
$$

A typical example is the function $p_{v}(\boldsymbol{x}, r)=r_{+}$for all $r \in \mathbb{R}$, a.e. $\boldsymbol{x} \in \Gamma_{3}$, where $r_{+}$denotes the positive part of $r$. Then, for every penalty parameter $\lambda>0$, we consider the following frictionless contact problem without unilateral constraint.

Problem 33 Find a displacement field $\boldsymbol{u}_{\lambda}: \Omega \rightarrow \mathbb{R}^{d}$, a stress field $\sigma_{\lambda}: \Omega \rightarrow \mathbb{S}^{d}$ and an interface force $\xi_{\lambda v}: \Gamma_{3} \rightarrow \mathbb{R}$ such that

$$
\begin{aligned}
& \boldsymbol{\sigma}_{\lambda}=\mathcal{F} \boldsymbol{\varepsilon}\left(\boldsymbol{u}_{\lambda}\right) \quad \text { in } \Omega, \\
& \operatorname{Div} \boldsymbol{\sigma}_{\lambda}+\boldsymbol{f}_{0}=\mathbf{0} \quad \text { in } \Omega, \\
& \boldsymbol{u}_{\lambda}=\mathbf{0} \quad \text { on } \Gamma_{1}, \\
& \boldsymbol{\sigma}_{\lambda} \boldsymbol{v}=\boldsymbol{f}_{2} \quad \text { on } \Gamma_{2}, \\
& -\sigma_{\lambda v}=\frac{1}{\lambda} p_{v}\left(u_{\lambda v}-g\right)+\xi_{\lambda v}, \quad \xi_{\lambda v} \in \partial j_{v}\left(u_{\lambda v}\right) \text { on } \Gamma_{3}, \\
& -\boldsymbol{\sigma}_{\lambda \tau}=\mathbf{0} \text { on } \Gamma_{3} .
\end{aligned}
$$

Here and below $u_{\lambda v}$ and $\sigma_{\lambda v}$ denote the normal components of the unknowns $\boldsymbol{u}_{\lambda}$ and $\sigma_{\lambda}$, and $\sigma_{\lambda \tau}$ represents the tangential part of the tensor $\sigma_{\lambda}$, respectively. Also, $\lambda$ may be interpreted as a deformability coefficient of the foundation, and then $\frac{1}{\lambda}$ is the surface stiffness coefficient.

Next, we use the Riesz representation theorem to define the operator $P: V \rightarrow V^{*}$ by

$$
\langle P \boldsymbol{u}, \boldsymbol{v}\rangle=\int_{\Gamma_{3}} p_{v}\left(u_{v}-g\right) v_{v} d \Gamma \quad \text { for all } \boldsymbol{u}, \boldsymbol{v} \in V .
$$


Then, the variational formulation of Problem 33, in terms of displacement, is the following.

Problem 34 Find a displacement field $\boldsymbol{u}_{\lambda} \in V$ such that

$$
\begin{aligned}
& \left(\mathcal{F} \boldsymbol{\varepsilon}\left(\boldsymbol{u}_{\lambda}\right), \boldsymbol{\varepsilon}(\boldsymbol{v})-\boldsymbol{\varepsilon}\left(\boldsymbol{u}_{\lambda}\right)\right)_{\mathcal{H}}+\frac{1}{\lambda}\left\langle P \boldsymbol{u}_{\lambda}, \boldsymbol{v}-\boldsymbol{u}_{\lambda}\right\rangle \\
& \quad+\int_{\Gamma_{3}} j_{v}^{0}\left(u_{\lambda v} ; v_{v}-u_{\lambda v}\right) d \Gamma \geq\left\langle\boldsymbol{f}, \boldsymbol{v}-\boldsymbol{u}_{\lambda}\right\rangle \quad \text { for all } \boldsymbol{v} \in V .
\end{aligned}
$$

Note also that, since we assume (74), Problem 31 can be reformulated in a simplified way, as follows.

Problem 35 Find a displacement field $\boldsymbol{u} \in U$ such that

$$
(\mathcal{F} \boldsymbol{\varepsilon}(\boldsymbol{u}), \boldsymbol{\varepsilon}(\boldsymbol{v})-\boldsymbol{\varepsilon}(\boldsymbol{u}))_{\mathcal{H}}+\int_{\Gamma_{3}} j_{v}^{0}\left(u_{v} ; v_{v}-u_{v}\right) d \Gamma \geq\langle\boldsymbol{f}, \boldsymbol{v}-\boldsymbol{u}\rangle \quad \text { for all } \boldsymbol{v} \in U .
$$

We have the following existence, uniqueness and convergence result.

Theorem 36 Assume (64), (66), (67) and (75) and, moreover, assume that

$$
\alpha_{j_{v}} c_{k}^{2}\|\gamma\|^{2}<m_{\mathcal{F}}
$$

either $j_{v}(\boldsymbol{x}, \cdot)$ or $-j_{v}(\boldsymbol{x}, \cdot)$ is regular on $\mathbb{R}$ for a.e. $\boldsymbol{x} \in \Gamma_{3}$.

Then

(i) there exists a unique solution $\boldsymbol{u} \in U$ to Problem 35;

(ii) for each $\lambda>0$, there exists a unique solution $\boldsymbol{u}_{\lambda} \in V$ to Problem 34;

(iii) $\boldsymbol{u}_{\lambda} \rightarrow \boldsymbol{u}$ in $V$, as $\lambda \rightarrow 0$.

Proof (i). The unique solvability of Problem 35 is a direct consequence of Theorem 32 since condition (70) is guaranteed by assumption (83) and $L_{F_{b}}=0$.

(ii) and (iii). We use Lemma 5.7 in [31] to see that assumption (75) on the function $p_{v}$ implies that the operator $P$ defined by (82) is monotone, Lipschitz continuous and $P \boldsymbol{v}=\mathbf{0}_{V^{*}}$ if and only if $v \in U$. Therefore, it follows from Definition 25 that $P$ is a penalty operator of $U$. On the other hand, the compactness of the trace operator and assumption (84) allow to use Lemma 29 with $X=V, Y=L^{2}\left(\Gamma_{3} ; \mathbb{R}^{d}\right), L=\gamma, \boldsymbol{v}_{0}=\mathbf{0}_{L^{2}\left(\Gamma_{3} ; \mathbb{R}^{d}\right)}$ and

$$
j(\boldsymbol{v})=\int_{\Gamma_{3}} j_{v}\left(\boldsymbol{x}, v_{v}(\boldsymbol{x})\right) d \Gamma \quad \text { for all } \boldsymbol{v} \in L^{2}\left(\Gamma_{3} ; \mathbb{R}^{d}\right) .
$$

As a result, we deduce that the function $j$ satisfies conditions (24) and (44). Moreover, condition (43) is obviously satisfied since, in our case, $\varphi \equiv 0$. We are now in a position to use Theorem 28 in order to conclude the proof of the theorem.

In addition to the mathematical interest in the convergence result in Theorem 36 (iii), it is important from the mechanical point of view, since it shows that the weak solution of the elastic frictionless contact problem with a deformable foundation approaches, as closely as one wishes, by the solution of an elastic frictionless contact problem with a rigid-deformable foundation, with a sufficiently small coefficient of deformability of the foundation. 
Open Access This article is distributed under the terms of the Creative Commons Attribution 4.0 International License (http://creativecommons.org/licenses/by/4.0/), which permits unrestricted use, distribution, and reproduction in any medium, provided you give appropriate credit to the original author(s) and the source, provide a link to the Creative Commons license, and indicate if changes were made.

\section{References}

1. Baiocchi, C., Capelo, A.: Variational and Quasivariational Inequalities: Applications to Free-Boundary Problems. Wiley, Chichester (1984)

2. Brézis, H.: Equations et inéquations non linéaires dans les espaces vectoriels en dualité. Ann. Inst. Fourier (Grenoble) 18, 115-175 (1968)

3. Brézis, H.: Problèmes unilatéraux. J. Math. Pures Appl. 51, 1-168 (1972)

4. Browder, F.E.: Nonlinear monotone operators and convex sets in Banach spaces. Bull. Am. Math. Soc. 71, 780-785 (1965)

5. Carl, S.: Barrier solutions of elliptic variational inequalities. Nonlinear Anal., Real World Appl. 26, 75-92 (2015)

6. Carl, S., Le, V.K.: Elliptic inequalities with multivalued operators: existence, comparison and related variational-hemivariational type inequalities. Nonlinear Anal., Theory Methods Appl. 121, 130-152 (2015)

7. Clarke, F.H.: Optimization and Nonsmooth Analysis. Wiley-Interscience, New York (1983)

8. Denkowski, Z., Migórski, S., Papageorgiou, N.S.: An Introduction to Nonlinear Analysis: Theory. Kluwer Academic/Plenum, Boston (2003)

9. Denkowski, Z., Migórski, S., Papageorgiou, N.S.: An Introduction to Nonlinear Analysis: Applications. Kluwer Academic/Plenum, Boston (2003)

10. Eck, C., Jarušek, J., Krbeč, M.: Unilateral Contact Problems: Variational Methods and Existence Theorems. Pure and Applied Mathematics, vol. 270. Chapman/CRC Press, New York (2005)

11. Han, W., Migórski, S., Sofonea, M.: A class of variational-hemivariational inequalities with applications to frictional contact problems. SIAM J. Math. Anal. 46, 3891-3912 (2014)

12. Han, W., Sofonea, M.: Quasistatic Contact Problems in Viscoelasticity and Viscoplasticity. Studies in Advanced Mathematics, vol. 30. Americal Mathematical Society/International Press, Providence/Somerville (2002)

13. Haslinger, J., Miettinen, M., Panagiotopoulos, P.D.: Finite Element Method for Hemivariational Inequalities. Theory, Methods and Applications. Kluwer Academic, Boston (1999)

14. Hlaváček, I., Haslinger, J., Necǎs, J., Lovíšek, J.: Solution of Variational Inequalities in Mechanics. Springer, New York (1988)

15. Khan, A.A., Motreanu, D.: Existence theorems for elliptic and evolutionary variational and quasivariational inequalities. J. Optim. Theory Appl. 167, 1136-1161 (2015)

16. Kikuchi, N., Oden, J.T.: Contact Problems in Elasticity: A Study of Variational Inequalities and Finite Element Methods. SIAM, Philadelphia (1988)

17. Kinderlehrer, D., Stampacchia, G.: An Introduction to Variational Inequalities and their Applications. Classics in Applied Mathematics, vol. 31. SIAM, Philadelphia (2000)

18. Lions, J.L., Stampacchia, G.: Variational inequalities. Commun. Pure Appl. Math. 20, 493-519 (1967)

19. Liu, Z.H., Zeng, B.: Optimal control of generalized quasi-variational hemivariational inequalities and its applications. Appl. Math. Optim. 72, 305-323 (2015)

20. Martins, J.A.C., Monteiro Marques, M.D.P. (eds.): Contact Mechanics. Kluwer, Dordrecht (2002)

21. Migórski, S., Ochal, A., Sofonea, M.: Nonlinear Inclusions and Hemivariational Inequalities. Models and Analysis of Contact Problems. Advances in Mechanics and Mathematics, vol. 26. Springer, New York (2013)

22. Migórski, S., Ochal, A., Sofonea, M.: History-dependent variational-hemivariational inequalities in contact mechanics. Nonlinear Anal., Real World Appl. 22, 604-618 (2015)

23. Naniewicz, Z., Panagiotopoulos, P.D.: Mathematical Theory of Hemivariational Inequalities and Applications. Marcel Dekker/Basel, New York/Hong Kong (1995)

24. Panagiotopoulos, P.D.: Nonconvex problems of semipermeable media and related topics. Z. Angew. Math. Mech. 65, 29-36 (1985)

25. Panagiotopoulos, P.D.: Inequality Problems in Mechanics and Applications. Birkhäuser, Boston (1985)

26. Panagiotopoulos, P.D.: Hemivariational Inequalities, Applications in Mechanics and Engineering. Springer, Berlin (1993)

27. Pascali, D., Sburlan, S.: Nonlinear Mappings of Monotone Type. Sijthoff and Noordhoff, Alpen aan den Rijn (1978) 
28. Shillor, M. (ed.): Recent advances in contact mechanics, Special issue. Math. Comput. Modelling 28 (4-8) (1998)

29. Shillor, M., Sofonea, M., Telega, J.J.: Models and Analysis of Quasistatic Contact. Lect. Notes Phys., vol. 655. Springer, Berlin (2004)

30. Sofonea, M., Han, W., Migórski, S.: Numerical analysis of history-dependent variational inequalities with applications to contact problems. Eur. J. Appl. Math. 26, 427-452 (2015)

31. Sofonea, M., Matei, A.: Mathematical Models in Contact Mechanics. London Mathematical Society Lecture Note Series, vol. 398. Cambridge Univ. Press, London (2012)

32. Tang, G.J., Huang, N.J.: Existence theorems of the variational-hemivariational inequalities. J. Glob. Optim. 56, 605-622 (2013)

33. Zeidler, E.: Nonlinear Functional Analysis and Applications II A/B. Springer, New York (1990) 